\title{
Synthesis of Morpholin-2-ones by Chemoselective Intramolecular Rhodium- Catalyzed Reductive Ring Expansion of Oxazolidines
}

\author{
Maksym Vasylyev and Howard Alper* \\ Contribution from the Centre for Catalysis Research and Innovation, Department of Chemistry, \\ University of Ottawa, 10 Marie Curie, Ottawa, Ontario, Canada K1N 6N5 \\ e-mail: howard.alper@uottawa.ca

\section{Supporting Information}

\section{Table of contents}

1. General Information

2. General Procedure for the Synthesis of N-(Ethoxycarboxymethyl)oxazolidines 1a-g and their Characterization Data.

3. General Procedure for the Catalytic Ring Expansion Reaction and Characterization Data for 4- $N$-Methylmorpholin-2-ones 3a-g

4. ${ }^{1} \mathrm{H}$ and ${ }^{13} \mathrm{C}$ NMR Spectra

5. Chiral GC (compounds $\mathbf{3 c}$ and $\mathbf{3 d}$ and their mixture) 


\section{General Information}

Ethyl bromoacetate, paraformaldehyde, potassium iodide, L-phenylalanine, D-phenylalanine, Lleucine, and D-valine were obtained from Aldrich and used as received. Sodium borohydride and chloro(1,5-cyclooctadiene)rhodium(I) dimer were obtained from Strem and used as received. Iodine, Lvaline, L-phenylglycine, and D-phenylglycine were obtained from Alfa Aesar and used as received.

Chiral amino alcohols were prepared according to the published literature procedure. ${ }^{1}$ Hydrogen gas and carbon monoxide were purchased from BOC Gases and were used as received. ${ }^{2,3}$ All reactions were carried out in flame-dried glassware. Tetrahydrofuran was distilled from sodium immediately prior to use. Toluene was distilled from sodium metal. Flash chromatography was performed on silica gel $60 \AA$ (Aldrich, 200-425 mesh) with the indicated eluent. High-resolution mass spectra were recorded on VG $7070 \mathrm{E}$ spectrometer. ${ }^{1} \mathrm{H} \mathrm{NMR}$ and ${ }^{13} \mathrm{C} \mathrm{NMR}$ spectra were recorded at ambient temperature in $\mathrm{CDCl}_{3}$ on a Bruker Avance 300 spectrometer at 300.13 and $75.47 \mathrm{MHz}$ respectively. The chemical shifts are reported in ppm relative to an internal tetramethylsilane standard.

Optical rotationss $(\alpha)$ were measured at the sodium D line using a $1 \mathrm{~mL}$ cell with a $1 \mathrm{dm}$ path length on a Perkin-Elmer 241 polarimeter. For the $[\alpha]^{T}{ }_{D}$ the concentration $c$ is given in $\mathrm{g} / 100 \mathrm{~mL}$.

\footnotetext{
${ }^{1}$ McKennon, J. M.; Meyers A. I. J. Org. Chem. 1993, 58, 3568 - 3571.

2 Due to the explosive properties of hydrogen gas it should be used only in a specially designed laboratory equipped with protected spark-free electrical equipment inside a well-ventilated fume hood.

${ }^{3}$ Carbon monoxide is a highly poisonous gas, which should be used with extreme care in a wellventilated fume hood in the laboratory equipped with $\mathrm{CO}$ detectors.
} 


\section{General Procedure for the Synthesis of $N$-(Ethoxycarboxymethyl)oxazolidines 1a-g}

In a round-bottom flask quipped with a magnetic stirring bar was added $16.4 \mathrm{mmol}$ of amino alcohol and100 $\mathrm{mL}$ of anhydrous THF under an argon atmosphere. To this solution were added $3.15 \mathrm{~mL}(18.1 \mathrm{mmol})$ of diiso-propyl ethylamine and $1.94 \mathrm{~mL}(17.5 \mathrm{mmol})$ of ethyl bromoacetate. The reaction mixture was stirred for $48 \mathrm{hrs}$ at room temperature. The solution of the alkylated amino alcohol was then separated from precipitated di-iso-propyl ethylammonium bromide by filtration through a short plug of silica. All volatiles were evaporated off and to the oily residue $0.5 \mathrm{~g}$ of paraformaldehyde was added followed by $100 \mathrm{~mL}$ of anhydrous toluene. The flask was then connected to a Soxhlet apparatus with a thimble containing $\mathrm{CaH}_{2}$. The reaction mixture was refluxed for $5 \mathrm{hrs}$, cooled and concentrated on a rotary evaporator. Purification of the crude product was performed by flash chromatography using the indicated eluant.

\section{(4R)- $N$-(Ethoxycarboxymethyl)-4-phenyloxazolidine (1a):}

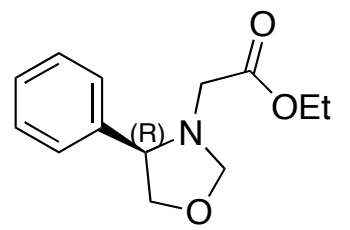

The crude compound was purified by flash chromatography with hexanes/EtOAc (1:1)

mixture used as eluant to obtain the title compound as pale-yellow oil in $87 \%$ yield. $\mathrm{R}_{f}=0.57 ;{ }^{1} \mathrm{H}$ NMR $\delta \mathrm{ppm} 1.2(\mathrm{t}, J=7.1 \mathrm{~Hz}, 3 \mathrm{H}), 3.3(\mathrm{~d}, J=16.8 \mathrm{~Hz}, 1 \mathrm{H}), 3.5(\mathrm{~d}, 1$ H), 3.7 (t, $J=7.8 \mathrm{~Hz}, 1 \mathrm{H}), 4.0$ (t, $J=7.4 \mathrm{~Hz}, 1 \mathrm{H}), 4.1(\mathrm{q}, J=7.2 \mathrm{~Hz}, 1 \mathrm{H}), 4.3(\mathrm{dd}, J$ $=7.8, J=7.1 \mathrm{~Hz}, 1 \mathrm{H}), 4.4(\mathrm{~d}, J=3.3 \mathrm{~Hz}, 1 \mathrm{H}), 4.9(\mathrm{~d}, J=3.3 \mathrm{~Hz}, 1 \mathrm{H}), 7.4(\mathrm{~m}, 5 \mathrm{H}) ;{ }^{13} \mathrm{C} \mathrm{NMR} \delta \mathrm{ppm}$ $14.2,52.9,60.8,66.7,73.4,86.9,127.7,128.0,128.7,138.9,170.6$; HRMS (EI) Calcd. for $\mathrm{C}_{13} \mathrm{H}_{17} \mathrm{NO}_{3}$ 235.1209, found 235.1204; $[\alpha]_{\mathrm{D}}^{22}-128.3(c 1.27, \mathrm{DCM})$.

\section{(4S)- $N$-(Ethoxycarboxymethyl)-4-phenyloxazolidine (1b):}

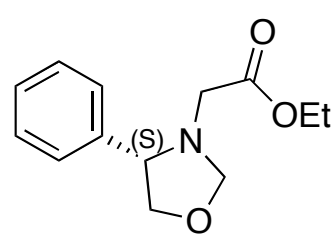

The crude compound was purified by flash chromatography with hexanes/EtOAc (1:1) mixture used as eluant to obtain the title compound as pale-yellow oil in $93 \%$ yield. $\mathrm{R}_{f}=0.57 ;{ }^{1} \mathrm{H}$ NMR $\delta$ ppm $1.2(\mathrm{t}, J=7.1 \mathrm{~Hz}, 3 \mathrm{H}), 3.3(\mathrm{~d}, J=16.8 \mathrm{~Hz}, 1 \mathrm{H}), 3.5(\mathrm{~d}, J$ $=16.8 \mathrm{~Hz}, 1 \mathrm{H}), 3.7(\mathrm{t}, J=7.8 \mathrm{~Hz}, 1 \mathrm{H}), 4.0(\mathrm{t}, J=7.4 \mathrm{~Hz}, 1 \mathrm{H}), 4.1$ (q, $J=7.2 \mathrm{~Hz}, 2$ H), $4.3(\mathrm{t}, \mathrm{J}=7.1,1 \mathrm{H}), 4.4(\mathrm{~d}, J=3.3 \mathrm{~Hz}, 1 \mathrm{H}), 4.9(\mathrm{~d}, J=3.3 \mathrm{~Hz}, 1 \mathrm{H}), 7.4(\mathrm{~m}, 5 \mathrm{H}) ;{ }^{13} \mathrm{C}$ NMR $\delta \mathrm{ppm}$ $14.2,53.0,60.8,66.8,73.4,86.9,127.7,128.0,128.8,138.9,170.6$; HRMS (EI) Calcld. for $\mathrm{C}_{13} \mathrm{H}_{17} \mathrm{NO}_{3}-$ 235.1209 , found $-235.1195 ;[\alpha]_{D}^{22}+129.7(c 1.65, \mathrm{DCM})$. 
(4R)-N-(Ethoxycarboxymethyl)-4-isobutyloxazolidine (1c):

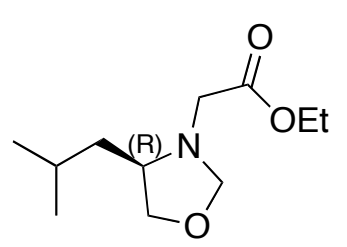

The crude compound was purified by flash chromatography with $20 \%$ of EtOAc in hexanes used as eluant to obtain the title compound as colorless oil in $82 \%$ yield.

$\mathrm{R}_{f}=0.53 ;{ }^{1} \mathrm{H}$ NMR $\delta$ ppm $0.9(\mathrm{~d}, J=6.6 \mathrm{~Hz}, 6 \mathrm{H}), 1.2(\mathrm{~m}, 1 \mathrm{H}), 1.3(\mathrm{t}, J=7.2 \mathrm{~Hz}, 3$

H), $1.6(\mathrm{~m}, 2 \mathrm{H}), 3.1(\mathrm{~m}, 1 \mathrm{H}), 3.3(\mathrm{dd}, J=7.9, J=5.8 \mathrm{~Hz}, 1 \mathrm{H}), 3.4(\mathrm{~d}, J=6.0 \mathrm{~Hz}, 2$ H), $4.0(\mathrm{dd}, J=7.9, J=6.9 \mathrm{~Hz}, 1 \mathrm{H}), 4.2(\mathrm{q}, J=7.1 \mathrm{~Hz}, 2 \mathrm{H}), 4.3(\mathrm{~d}, J=5.7 \mathrm{~Hz}, 1 \mathrm{H}), 4.5(\mathrm{~d}, J=5.7 \mathrm{~Hz}, 1$ $\mathrm{H}) ;{ }^{13} \mathrm{C}$ NMR $\delta$ ppm 14.3, 22.8, 23.0, 25.5, 42.7, 56.0, 60.9, 62.2, 69.8, 86.3, 170.9; HRMS (EI) Calcld. for $\mathrm{C}_{11} \mathrm{H}_{21} \mathrm{NO}_{3}-215.1522$, Found. - 215.1525; $[\alpha]^{22}{ }_{\mathrm{D}}-32.5$ (c 1.17, DCM).

$(4 S)-N-($ Ethoxycarboxymethyl)-4-isobutyloxazolidine (1d):

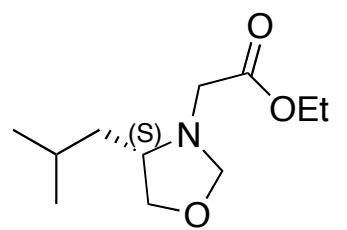

The crude compound was purified by flash chromatography with $20 \%$ of EtOAc in hexanes used as eluant to obtain the title compound as colorless oil in $90 \%$ yield.

$\mathrm{R}_{f}=0.53 ;{ }^{1} \mathrm{H}$ NMR $\delta$ ppm $0.9(\mathrm{~d}, J=6.6 \mathrm{~Hz}, 6 \mathrm{H}), 1.2(\mathrm{~m}, 1 \mathrm{H}), 1.3(\mathrm{t}, J=7.1 \mathrm{~Hz}, 3$ H), $1.6(\mathrm{~m}, 2 \mathrm{H}), 3.1(\mathrm{~m}, 1 \mathrm{H}), 3.3(\mathrm{dd}, J=7.9, J=5.8 \mathrm{~Hz}, 1 \mathrm{H}), 3.4(\mathrm{~d}, J=6.0 \mathrm{~Hz}, 2$ H) $4.0(\mathrm{dd}, J=7.9, J=6.9 \mathrm{~Hz}, 1 \mathrm{H}), 4.2(\mathrm{q}, J=7.1 \mathrm{~Hz}, 2 \mathrm{H}), 4.3(\mathrm{~d}, J=5.7 \mathrm{~Hz}, 1 \mathrm{H}), 4.5(\mathrm{~d}, J=5.7 \mathrm{~Hz}, 1$ $\mathrm{H}) ;{ }^{13} \mathrm{C}$ NMR $\delta$ ppm 14.3, 22.7, 23.0, 25.5, 42.6, 55.9, 60.9, 62.2, 69.8, 86.2,170.9; HRMS (EI) Calcld. for $\mathrm{C}_{11} \mathrm{H}_{21} \mathrm{NO}_{3}-215.1522$, Found $-215.1525 ;[\alpha]^{22}{ }_{\mathrm{D}}+31.0(c 1.84, \mathrm{DCM})$.

\section{(4R)- $N$-(Ethoxycarboxymethyl)-4-benzyloxazolidine (1e):}

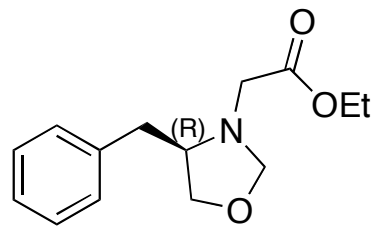

The crude compound was purified by flash chromatography with hexanes/EtOAc $(1: 1)$ mixture used as eluant to obtain the title compound as pale-yellow oil in $96 \%$ yield.

$\mathrm{R}_{f}=0.44 ;{ }^{1} \mathrm{H}$ NMR $\delta$ ppm $1.3(\mathrm{t}, J=7.1 \mathrm{~Hz}, 3 \mathrm{H}), 2.6(\mathrm{dd}, J=13.5, J=8.7 \mathrm{~Hz}, 1$ H), $3.0(\mathrm{dd}, J=13.5, J=5.9 \mathrm{~Hz}, 1 \mathrm{H}), 3.3(\mathrm{~m}, 1 \mathrm{H}), 3.4(\mathrm{~d}, J=5.7 \mathrm{~Hz}, 2 \mathrm{H}), 3.5(\mathrm{dd}, J=8.3, J=5.7 \mathrm{~Hz}, 1$ H), $3.9(\mathrm{dd}, J=8.3, J=6.8 \mathrm{~Hz}, 1 \mathrm{H}), 4.2(\mathrm{q}, J=7.1 \mathrm{~Hz}, 2 \mathrm{H}), 4.4(\mathrm{~d}, J=5.3 \mathrm{~Hz}, 1 \mathrm{H}), 4.6(\mathrm{~d}, J=5.3 \mathrm{~Hz}, 1$ H), 7.2 (m, 5 H); ${ }^{13} \mathrm{C}$ NMR $\delta$ ppm 14.2, 39.6, 55.7, 60.7, 64.9, 69.4, 86.4, 126.4, 128.4, 129.1, 138.4, 170.7; HRMS (EI) Calcld. for $\mathrm{C}_{14} \mathrm{H}_{19} \mathrm{NO}_{3}-249.1365$, Found - 249.1337; $\left.\alpha\right]^{22}{ }_{\mathrm{D}}+10.4$ (c 1.64, DCM).

\section{(4S)- $\mathrm{N}$-(Ethoxycarboxymethyl)-4-benzyloxazolidine (1f):}

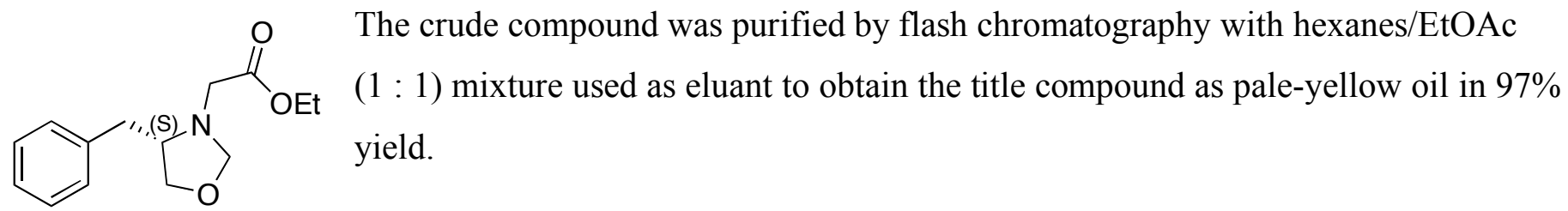


$\mathrm{R}_{f}=0.44 ;{ }^{1} \mathrm{H}$ NMR $\delta$ ppm $1.3(\mathrm{t}, J=7.2 \mathrm{~Hz}, 3 \mathrm{H}), 2.6(\mathrm{dd}, J=13.4, J=8.7 \mathrm{~Hz}, 1 \mathrm{H}), 3.0(\mathrm{dd}, J=13.5, J=$ $5.9 \mathrm{~Hz}, 1 \mathrm{H}), 3.3(\mathrm{~m}, 1 \mathrm{H}), 3.4(\mathrm{~d}, J=5.7 \mathrm{~Hz}, 2 \mathrm{H}), 3.5(\mathrm{dd}, J=8.3, J=5.7 \mathrm{~Hz}, 1 \mathrm{H}), 3.9(\mathrm{dd}, J=8.3, J=$ $6.8 \mathrm{~Hz}, 1 \mathrm{H}), 4.2(\mathrm{q}, J=7.1 \mathrm{~Hz}, 2 \mathrm{H}), 4.4(\mathrm{~d}, J=5.3 \mathrm{~Hz}, 1 \mathrm{H}), 4.6(\mathrm{~d}, J=5.3 \mathrm{~Hz}, 1 \mathrm{H}), 7.3(\mathrm{~m}, 5 \mathrm{H}) ;{ }^{13} \mathrm{C}$ NMR $\delta$ ppm 14.2, 39.7, 55.8, 60.8, 65.0, 69.5, 86.5, 126.4, 128.5, 129.1, 138.5, 170.7; HRMS (EI) Calcld. for $\mathrm{C}_{14} \mathrm{H}_{19} \mathrm{NO}_{3}-249.1365$ Found $-249.1340 ;[\alpha]_{D}^{22}-13.0$ (c 2.0, DCM).

\section{(4S)- $N$-(Ethoxycarboxymethyl)-4-isopropyloxazolidine (1g):}

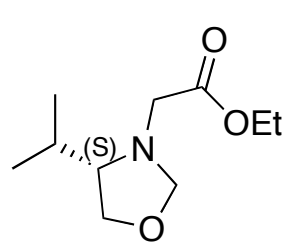

The crude compound was purified by flash chromatography with $20 \%$ of EtOAc in hexanes used as eluant to obtain the title compound as colorless oil in $79 \%$ yield.

$\mathrm{R}_{f}=0.36 ;{ }^{1} \mathrm{H}$ NMR $\delta$ ppm $0.8(\mathrm{~d}, J=6.7 \mathrm{~Hz}, 3 \mathrm{H}), 1.0(\mathrm{~d}, J=6.7 \mathrm{~Hz}, 3 \mathrm{H}), 1.2(\mathrm{t}, J=$ $7.1 \mathrm{~Hz}, 3 \mathrm{H}), 1.6(\mathrm{~m}, 1 \mathrm{H}), 2.6(\mathrm{~m}, 1 \mathrm{H}), 3.4(\mathrm{~m}, 3 \mathrm{H}), 3.9(\mathrm{dd}, J=8.2, J=7.4 \mathrm{~Hz}, 1 \mathrm{H})$,

4.1 (q, $J=7.1 \mathrm{~Hz}, 2 \mathrm{H}), 4.3$ (m, $2 \mathrm{H}) ;{ }^{13} \mathrm{C}$ NMR $\delta$ ppm 14.2, 18.6, 20.2, 31.4, 57.1, 60.7, 67.9, 70.4, 86.7, 171.0; HRMS (EI) Calcld. for $\mathrm{C}_{10} \mathrm{H}_{19} \mathrm{NO}_{3}-201.1365$, Found - 201.1349; $[\alpha]^{22}+16.7(c 1.42$, DCM).

\section{General Procedure for the Catalytic Ring Expansion Reaction}

In a typical procedure $1 \mathrm{mmol}$ of $\mathrm{N}$-(ethoxycarboxymethyl)oxazolidine and $0.05 \mathrm{mmol}$ of $[\mathrm{Rh}(\mathrm{COD}) \mathrm{Cl}]_{2}$ were dissolved in $10 \mathrm{~mL}$ of anhydrous toluene in a glass liner equipped with a magnetic stirring bar. To this solution was added $0.1 \mathrm{mmol}$ of $\mathrm{KI}$ and the liner was inserted into a $45 \mathrm{~mL}$ autoclave, which was then sealed. Carbon monoxide, 5 atm, was introduced to the autoclave by the two consecutive pump-release cycles. Finally, hydrogen gas was introduced into the autoclave bringing the overall pressure to $10 \mathrm{~atm}$. The autoclave was placed in a thermostated oil bath at $100{ }^{\circ} \mathrm{C}$ and the reaction was carried out for $15 \mathrm{~h}$. The autoclave was then cooled, and pressure was released. The reaction mixture was concentrated and the residue was subjected to flash chromatography using the indicated eluant.

\section{(5R)-4-N-Methyl-5-phenylmorpholin-2-one (3a):}

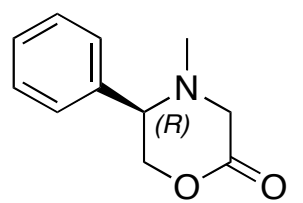

The title compound was isolated by flash chromatography with a mixture of EtOAc and hexanes $(1: 1)$ used as eluant to obtain it as colorless oil in $97 \%$ yield.

$\mathrm{R}_{f}=0.5 ;{ }^{1} \mathrm{H}$ NMR $\delta$ ppm $2.1(\mathrm{~s}, 3 \mathrm{H}), 3.1(\mathrm{~d}, J=17.8 \mathrm{~Hz}, 1 \mathrm{H}), 3.4(\mathrm{dd}, J=9.8, J=4.7$ $\mathrm{Hz}, 1 \mathrm{H}), 3.9(\mathrm{~d}, J=17.8 \mathrm{~Hz}, 1 \mathrm{H}), 4.3(\mathrm{~m}, 2 \mathrm{H}), 7.4(\mathrm{~m}, 5 \mathrm{H}) ;{ }^{13} \mathrm{C} \mathrm{NMR} \delta \mathrm{ppm}$ 42.6, 57.1, 64.8, 73.6, 128.4, 128.9, 129.1, 136.1, 167.8; HRMS (EI) Calcld. for $\mathrm{C}_{11} \mathrm{H}_{13} \mathrm{NO}_{2}-191.0947$, Found - 191.0937; $[\alpha]^{22}$ -59.5 (c 1.49, DCM). 


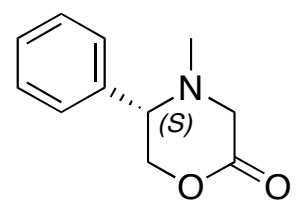

The title compound was isolated by flash chromatography with a mixture of EtOAc and hexanes $(1: 1)$ used as eluant to obtain it as colorless oil in $80 \%$ yield.

$\mathrm{R}_{f}=0.5 ;{ }^{1} \mathrm{H}$ NMR $\delta$ ppm $2.1(\mathrm{~s}, 3 \mathrm{H}), 3.2(\mathrm{~d}, J=17.8 \mathrm{~Hz}, 1 \mathrm{H}), 3.4(\mathrm{dd}, J=9.9, J=4.5$ $\mathrm{Hz}, 1 \mathrm{H}), 3.9(\mathrm{~d}, J=17.8 \mathrm{~Hz}, 1 \mathrm{H}), 4.3(\mathrm{~m}, 2 \mathrm{H}), 7.4(\mathrm{~m}, 5 \mathrm{H}) ;{ }^{13} \mathrm{C} \delta \mathrm{ppm} \mathrm{42.5,} \mathrm{56.9,}$ 64.7, 73.4, 128.2, 128.7, 129.0, 136.0, 167.6; HRMS (EI) Calcld. for $\mathrm{C}_{11} \mathrm{H}_{13} \mathrm{NO}_{2}-191.0947$, Found 191.0936; $[\alpha]_{\mathrm{D}}^{22}+60.3(c 1.41, \mathrm{DCM})$.

\section{(5R)-4-N-Methyl-5-isobutylmorpholin-2-one (3c):}

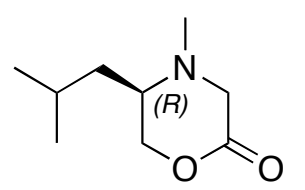

The title compound was isolated by flash chromatography with a mixture of EtOAc and hexanes $(1: 1)$ used as eluant to obtain it as colorless oil in $95 \%$ yield.

$\mathrm{R}_{f}=0.23 ;{ }^{1} \mathrm{H}$ NMR $\delta$ ppm $0.9(\mathrm{dd}, J=6.5, J=3.8 \mathrm{~Hz}, 6 \mathrm{H}), 1.2(\mathrm{~m}, 1 \mathrm{H}), 1.5(\mathrm{~m}, 1 \mathrm{H})$, $1.6(\mathrm{~m}, 1 \mathrm{H}), 2.3(\mathrm{~s}, 3 \mathrm{H}), 2.6(\mathrm{~m}, 1 \mathrm{H}), 3.2(\mathrm{~d}, J=17.8 \mathrm{~Hz}, 1 \mathrm{H}), 3.5(\mathrm{~d}, J=17.8 \mathrm{~Hz}, 1 \mathrm{H}), 4.1(\mathrm{dd}, J=11.1$, $J=8.7 \mathrm{~Hz}, 1 \mathrm{H}), 4.3(\mathrm{dd}, J=11.1, J=3.7 \mathrm{~Hz}, 1 \mathrm{H}) ;{ }^{13} \mathrm{C} \mathrm{NMR} \delta$ ppm 22.2, 23.5, 25.0, 36.8, 40.8, 55.7, 55.8, 71.5, 168.3; HRMS (EI) Calcld. for $\mathrm{C}_{9} \mathrm{H}_{17} \mathrm{NO}_{2}-171.1260$, Found: 171.1256; [ $\left.\alpha\right]^{22}{ }_{\mathrm{D}}-40.8$ (c 1.21, DCM).

\section{(5S)-4- $N$-Methyl-5-isobutylmorpholin-2-one (3d):}

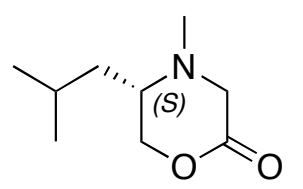

The title compound was isolated by flash chromatography with a mixture of EtOAc and hexanes $(1: 1)$ used as eluant to obtain it as colorless oil in $81 \%$ yield.

$\mathrm{R}_{f}=0.23 ;{ }^{1} \mathrm{H}$ NMR $\delta$ ppm $0.9(\mathrm{dd}, J=6.5, J=3.8 \mathrm{~Hz}, 6 \mathrm{H}), 1.2(\mathrm{~m}, 1 \mathrm{H}), 1.5(\mathrm{~m}, 1 \mathrm{H})$, $1.6(\mathrm{~m}, 1 \mathrm{H}), 2.3(\mathrm{~s}, 3 \mathrm{H}), 2.6(\mathrm{~m}, 1 \mathrm{H}), 3.2(\mathrm{~d}, J=17.8 \mathrm{~Hz}, 1 \mathrm{H}), 3.6(\mathrm{~d}, J=17.8 \mathrm{~Hz}, 1 \mathrm{H}), 4.1(\mathrm{dd}, J=11.1$, $J=8.6 \mathrm{~Hz}, 1 \mathrm{H}), 4.4(\mathrm{dd}, J=11.2, J=3.7 \mathrm{~Hz}, 1 \mathrm{H}) ;{ }^{13} \mathrm{C} \mathrm{NMR} \delta \mathrm{ppm} 22.2,23.6,25.1,36.8,40.8,55.7$, 55.9, 71.6, 168.3; HRMS (EI) Calcld. for $\mathrm{C}_{9} \mathrm{H}_{17} \mathrm{NO}_{2}-171.1260$, Found: 171.1248; $[\alpha]^{22}{ }_{\mathrm{D}}+38.3$ (c 1.84 , DCM).

(5R)-4-N-Methyl-5-benzylmorpholin-2-one (3e):

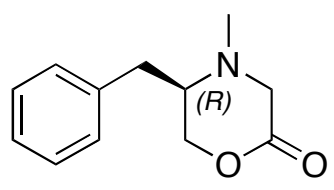

The title compound was isolated by flash chromatography with a mixture of EtOAc and hexanes $(1: 1)$ used as eluant to obtain it as pale-yellow oil in $97 \%$ yield.

$\mathrm{R}_{f}=0.32 ; 1 \mathrm{H}$ NMR $\delta$ ppm $2.4(\mathrm{~s}, 3 \mathrm{H}), 2.5(\mathrm{dd}, J=13.4, J=9.7 \mathrm{~Hz}, 1 \mathrm{H}), 2.8(\mathrm{~m}, 1$ 
H), $3.1(\mathrm{dd}, J=13.4, J=4.7 \mathrm{~Hz}, 1 \mathrm{H}), 3.3(\mathrm{~d}, J=17.7 \mathrm{~Hz}, 1 \mathrm{H}), 3.6(\mathrm{~d}, J=17.7 \mathrm{~Hz}, 1 \mathrm{H}), 4.1(\mathrm{~m}, 2 \mathrm{H}), 7.3$ (m, $5 \mathrm{H}) ;{ }^{13} \mathrm{C}$ NMR $\delta$ ppm 33.8, 41.6, 55.3, 59.2, 71.0, 126.9,128.8, 129.1, 137.2, 168.2; HRMS (EI) Calcld. for $\mathrm{C}_{12} \mathrm{H}_{15} \mathrm{NO}_{2}-205.1103$, Found: 205.1089; $[\alpha]^{22}{ }_{\mathrm{D}}+13.0(c$ 1.1, DCM).

\section{(5S)-4-N-Methyl-5-benzylmorpholin-2-one (3f):}

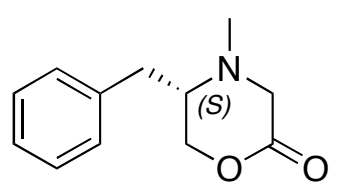

The title compound was isolated by flash chromatography with a mixture of EtOAc and hexanes $(1: 1)$ used as eluant to obtain it as pale-yellow oil in $81 \%$ yield.

$\mathrm{R}_{f}=0.32 ;{ }^{1} \mathrm{H}$ NMR $\delta$ ppm $2.4(\mathrm{~s}, 3 \mathrm{H}), 2.5(\mathrm{dd}, J=13.4, J=9.7 \mathrm{~Hz}, 1 \mathrm{H}), 2.8(\mathrm{~m}, 1$

H), $3.1(\mathrm{dd}, J=13.5, J=4.6 \mathrm{~Hz}, 1 \mathrm{H}), 3.3(\mathrm{~d}, J=17.8 \mathrm{~Hz}, 1 \mathrm{H}), 3.6(\mathrm{~d}, J=17.7 \mathrm{~Hz}, 1 \mathrm{H}), 4.1(\mathrm{~m}, 2 \mathrm{H}), 7.3$ (m, $5 \mathrm{H}) ;{ }^{13} \mathrm{C}$ NMR $\delta$ ppm 33.7, 41.6, 55.2, 59.2, 71.0, 126.8, 128.8, 129.1, 137.2, 168.1; HRMS (EI) Calcld. for $\mathrm{C}_{12} \mathrm{H}_{15} \mathrm{NO}_{2}-205.1103$, Found $-205.1070 ;[\alpha]_{D}^{22}-13.5$ (c 1.36, DCM).

\section{(5S)-4-N-Methyl-5-isopropylmorpholin-2-one (3g):}

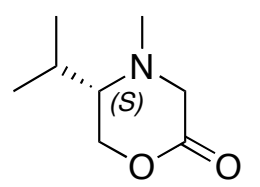

The title compound was isolated by flash chromatography with a mixture of EtOAc and hexanes $(1: 1)$ used as eluant to obtain it as pale-yellow oil in $76 \%$ yield.

$\mathrm{R}_{f}=0.25 ;{ }^{1} \mathrm{H}$ NMR $\delta$ ppm $0.9(\mathrm{~d}, J=6.9 \mathrm{~Hz}, 3 \mathrm{H}), 1.0(\mathrm{~d}, J=6.9 \mathrm{~Hz}, 3 \mathrm{H}), 2.0(\mathrm{~m}, 1 \mathrm{H})$, $2.3(\mathrm{~s}, 3 \mathrm{H}), 2.4(\mathrm{~m}, 1 \mathrm{H}), 3.2(\mathrm{~d}, J=17.1 \mathrm{~Hz}, 1 \mathrm{H}), 3.6(\mathrm{~d}, J=17.2 \mathrm{~Hz}, 1 \mathrm{H}), 4.2(\mathrm{dd}, J=11.4, J=9.9 \mathrm{~Hz}, 1$ H), 4.3 (dd, $J=10,4.4 \mathrm{~Hz}, 1 \mathrm{H}) ;{ }^{13} \mathrm{C}$ NMR $\delta$ ppm 16.9, 19.5, 27.9, 42.0, 56.0, 63.1, 68.2, 169.3; HRMS (EI) Calcld. for $\mathrm{C}_{8} \mathrm{H}_{15} \mathrm{NO}_{2}-157.1103$, Found: 157.1125; $[\alpha]^{22}{ }_{\mathrm{D}}-30.4(c 1.1, \mathrm{DCM})$.

\section{(4R)-N-(Cyanomethyl)-4-phenyloxazolidine* :}

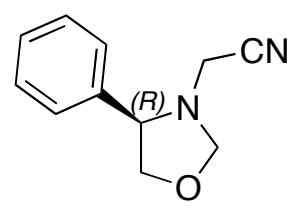

The title compound was isolated by flash chromatography with a mixture of EtOAc and hexanes $(1: 1)$ used as eluant to obtain it as pale-yellow oil in $83 \%$ yield.

$\mathrm{R}_{f}=0.58 ;{ }^{1} \mathrm{H}$ NMR $\delta$ ppm $3.5(\mathrm{~d}, J=17.5 \mathrm{~Hz}, 1 \mathrm{H}), 3.7(\mathrm{~d}, J=17.5 \mathrm{~Hz}, 1 \mathrm{H}), 3.8(\mathrm{t}, J$ $=8 \mathrm{~Hz}, 1 \mathrm{H}), 4.0(\mathrm{t}, J=8 \mathrm{~Hz}, 1 \mathrm{H}), 4.4(\mathrm{dd}, J=7.9, J=7.2 \mathrm{~Hz}, 1 \mathrm{H}), 4.5(\mathrm{~d}, J=2.8 \mathrm{~Hz}, 1 \mathrm{H}), 4.8(\mathrm{~d}, J=2.8$ $\mathrm{Hz}, 1 \mathrm{H}), 7.4$ (m, $5 \mathrm{H}) ;{ }^{13} \mathrm{C}$ NMR $\delta$ ppm 37.5, 65.4, 74.4, 85.4, 114.8, 127.7, 128.6, 129.0, 136.9; HRMS (EI) Calcld. for $\mathrm{C}_{11} \mathrm{H}_{12} \mathrm{~N}_{2} \mathrm{O}-188.0950$ Found 188.0945; $[\alpha]^{22}-240.1$ (c 2.19, DCM).

\footnotetext{
* The titled compound was synthesized according to the general procedure for the synthesis of $N$ (ethoxycarboxymethyl)oxazolidines, page S3. Bromoacetonotrile was used instead of ethyl bromoacetate.
} 

Solvent CHLOROFORM-D

Temperature (degree C) 27.000

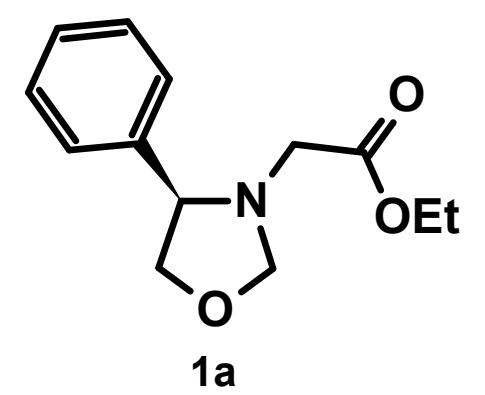

$1 \mathrm{a}$

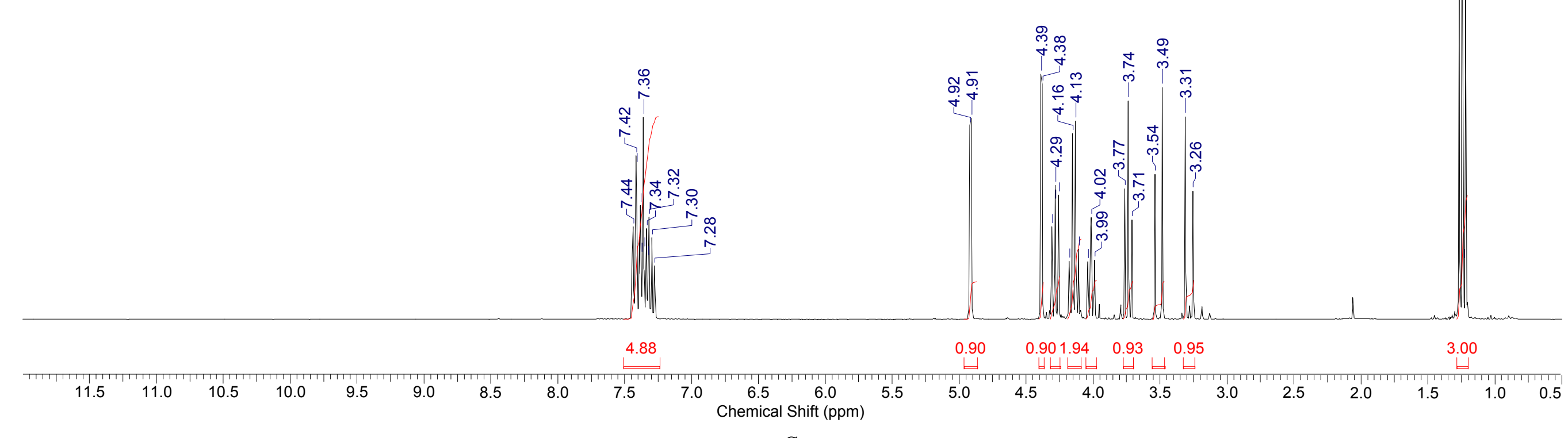




\begin{tabular}{|c|c|c|c|c|c|}
\hline Acquisition Time (sec) & \multirow{2}{*}{\multicolumn{3}{|c|}{\begin{tabular}{l|ll}
0.9110 & Comment & 13C with proton decoupling \\
& &
\end{tabular}}} & Date & 26 Apr 2007 19:59:00 \\
\hline File Name & C:IDocuments and Settings|Owner|DesktoplMAX_NMRIMax_HRO_015001r & & & Frequency $(\mathrm{MHz})$ & 75.47 \\
\hline Nucleus & $13 \mathrm{C}$ & Number of Transients 75 & \multirow{2}{*}{\begin{tabular}{l|l}
75 & Original Points Count \\
CHLOROFORM-D
\end{tabular}} & Points Count & 65536 \\
\hline Pulse Sequence & zgpg30 & Solvent & & Sweep Width $(\mathrm{Hz})$ & 17985.61 \\
\hline
\end{tabular}

Pulse Sequence zgpg30 Solvent CHLOROFORM-D

Sweep Width $(\mathrm{Hz})$

17985.61

Temperature (degree C) 27.000<smiles>CCOC(=O)CN1COC[C@H]1c1ccccc1</smiles>

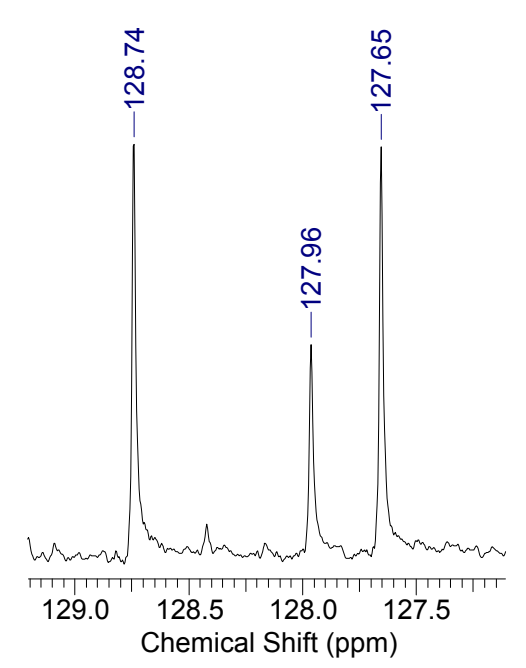

$128.74\rceil\ulcorner 127.65$

Chloroform-d

76.73

77.58

$86.89 \neg \quad-73.37$

66.7552 .93

$-60.83$

14.23

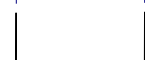

14.23

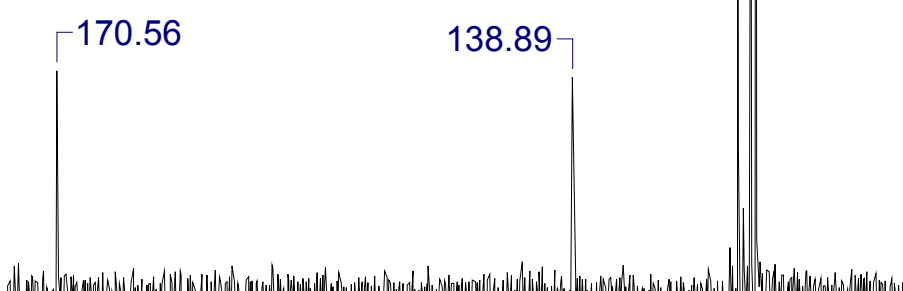

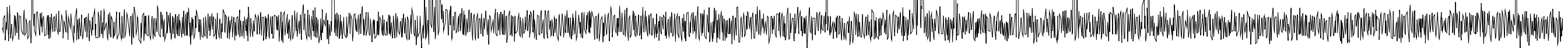
ताला।ाया 160 152 144 136 128 
\begin{tabular}{|ll|lc}
\hline Acquisition Time (sec) & 3.0228 & Date & 01 May 2007 21:19:00 \\
\hline File Name & C:IDocuments and SettingsIOwnerlDesktopIMAX_NMRIMax_HRO_016001r
\end{tabular}

Nucleus

Frequency $(\mathrm{MHz})$

Points Count

Pulse Sequence

Solvent CHLOROFORM-D

Sweep Width $(\mathrm{Hz})$

5081.30

Temperature (degree C) 27.000

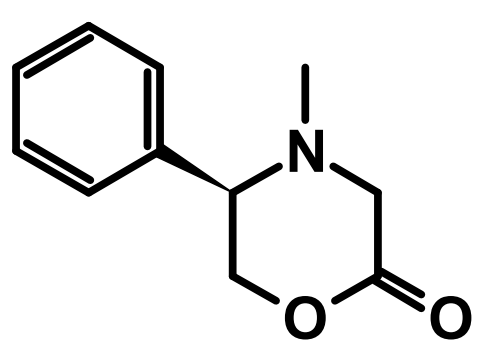

$3 a$

Chloroform-d

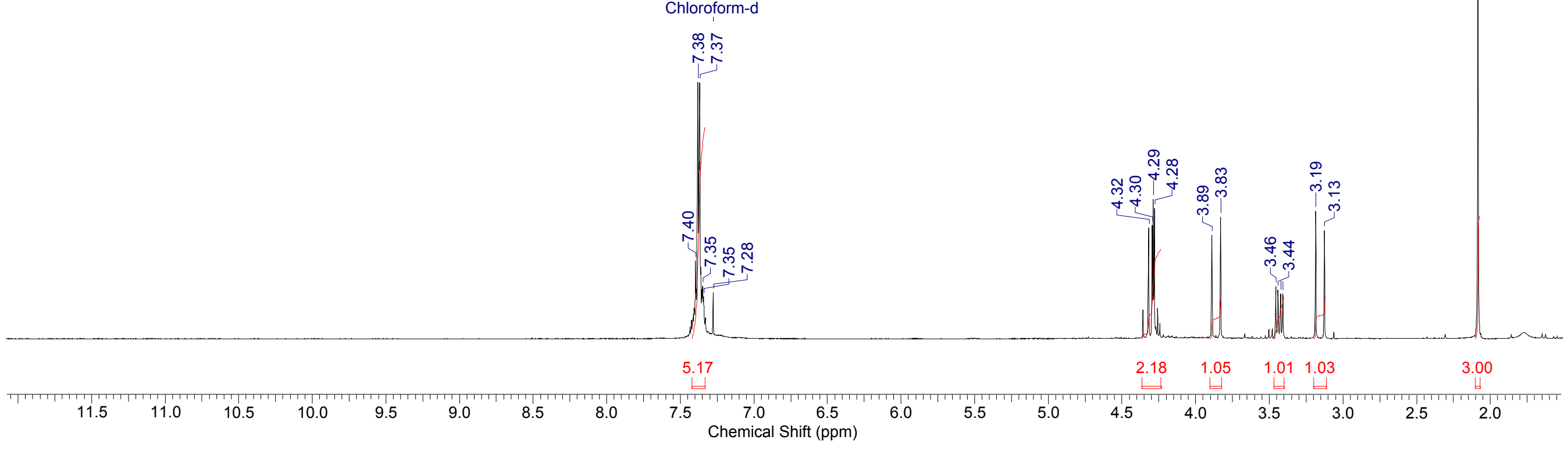




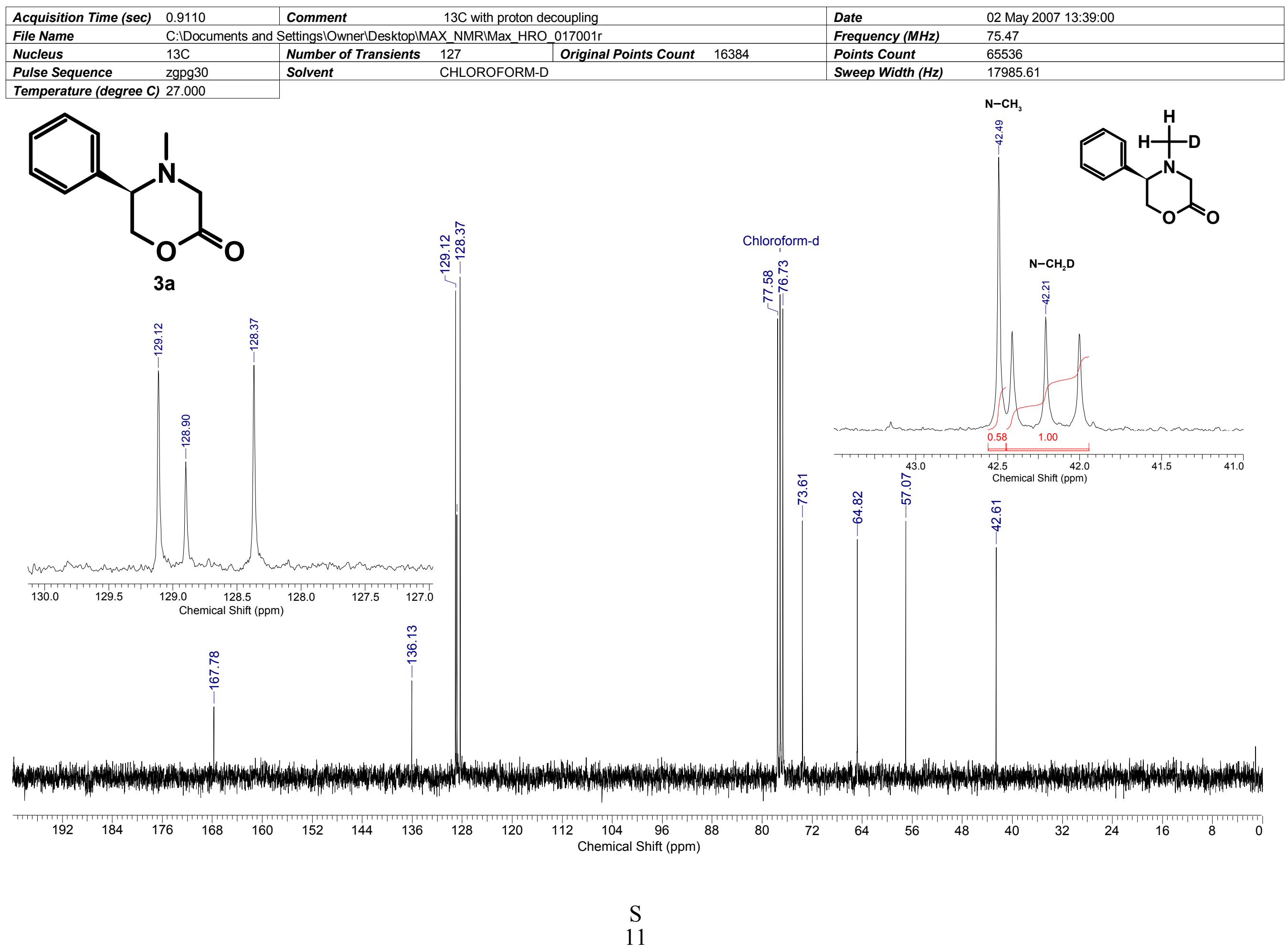


\begin{tabular}{|ll|lr}
\hline Acquisition Time (sec) & 3.0228 & Date & 25 Jul 2007 18:17:00
\end{tabular}

File Name C:IDocuments and SettingslOwnerlDesktopIMAX_NMRIMax_HRO_055001r

Number of Transients 16

Pulse Sequence $\quad \mathrm{zg} 30$

Original Points Count 15360

Frequency $(\mathrm{MHz})$

Points Count

300.13 Solvent

CHLOROFORM-D

Sweep Width $(\mathrm{Hz})$

Temperature (degree C) 27.000

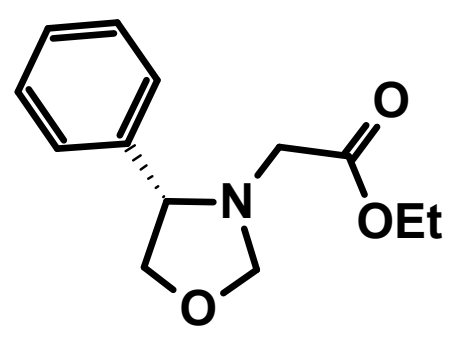

$1 \mathrm{~b}$

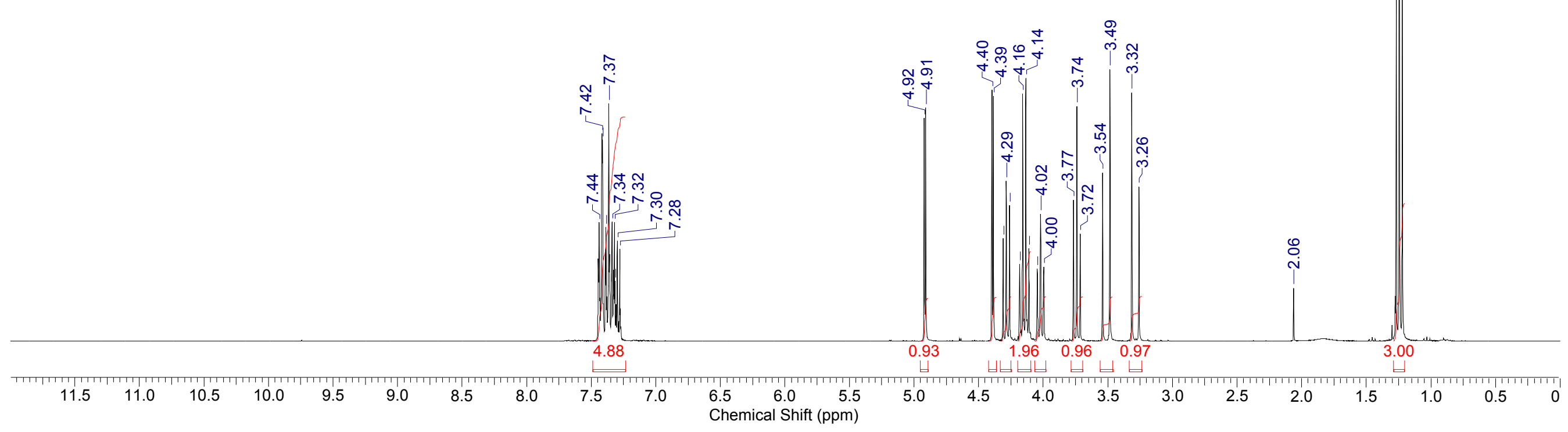




\begin{tabular}{|c|c|c|c|c|c|c|}
\hline Acquisition Time (sec) & 0.9110 & Comment & \multicolumn{2}{|c|}{$13 \mathrm{C}$ with proton decoupling } & Date & 25 Jul 2007 18:19:00 \\
\hline File Name & \multicolumn{4}{|c|}{ C:IDocuments and SettingsIOwnerlDesktopIMAX_NMRIMax_HRO_056001r } & Frequency $(\mathrm{MHz})$ & 75.47 \\
\hline Nucleus & $13 \mathrm{C}$ & Number of Transients & \multirow{2}{*}{\multicolumn{2}{|c|}{\begin{tabular}{l|l}
56 & Original Points Count \\
CHLOROFORM-D &
\end{tabular}}} & Points Count & 65536 \\
\hline Pulse Sequence & zgpg30 & Solvent & & & Sweep Width $(\mathrm{Hz})$ & 17985.61 \\
\hline
\end{tabular}

Pulse Sequence zgpg30 Solvent CHLOROFORM-D

Sweep Width $(\mathrm{Hz})$

Temperature (degree C) 27.000
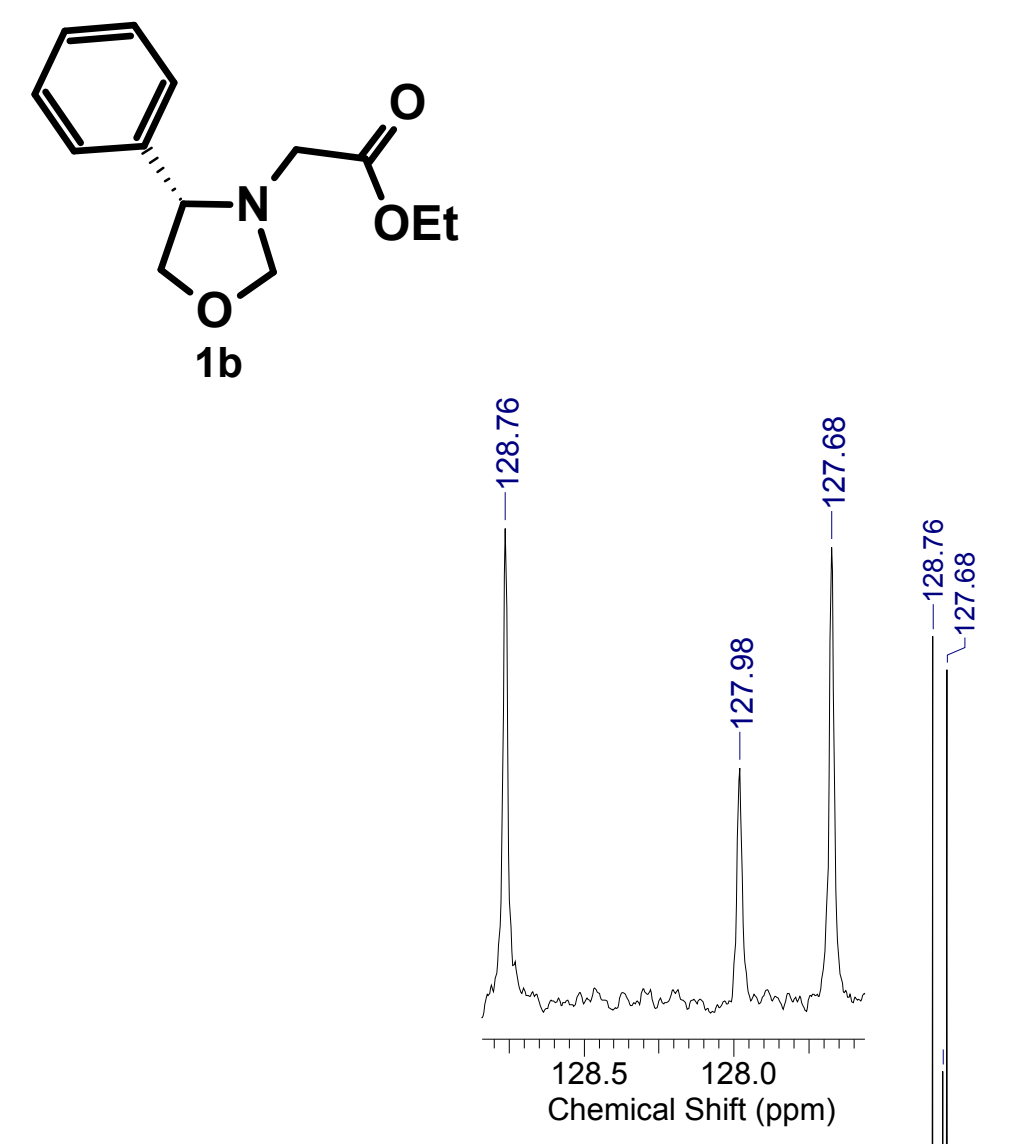

Chloroform-d

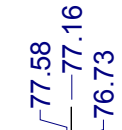

চூ.

Chemical Shift (ppm)

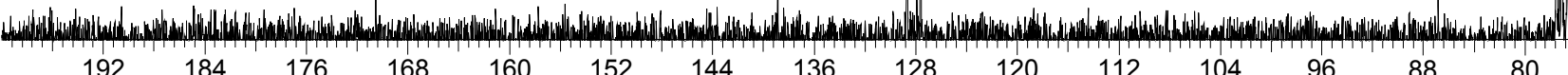


\begin{tabular}{|ll|lr}
\hline Acquisition Time (sec) & 3.0228 & Date & 27 Jul 2007 19:14:00 \\
\hline & C:I &
\end{tabular}

File Name $\quad$ C:IDocuments and SettingsiOwnerlDesktopIMAX_NMRIMax_HRO_059001r

\begin{tabular}{ll|l} 
Nucleus & $1 \mathrm{H}$ & Number of Transients 16
\end{tabular}

Original Points Count 15360

Frequency $(\mathrm{MHz})$

Points Count

300.13

Pulse Sequence $\mathrm{zg} 30$

Solvent

CHLOROFORM-D

Sweep Width $(\mathrm{Hz})$

Temperature (degree C) 27.000<smiles>CN1CC(=O)OC[C@H]1c1ccccc1</smiles>

3b

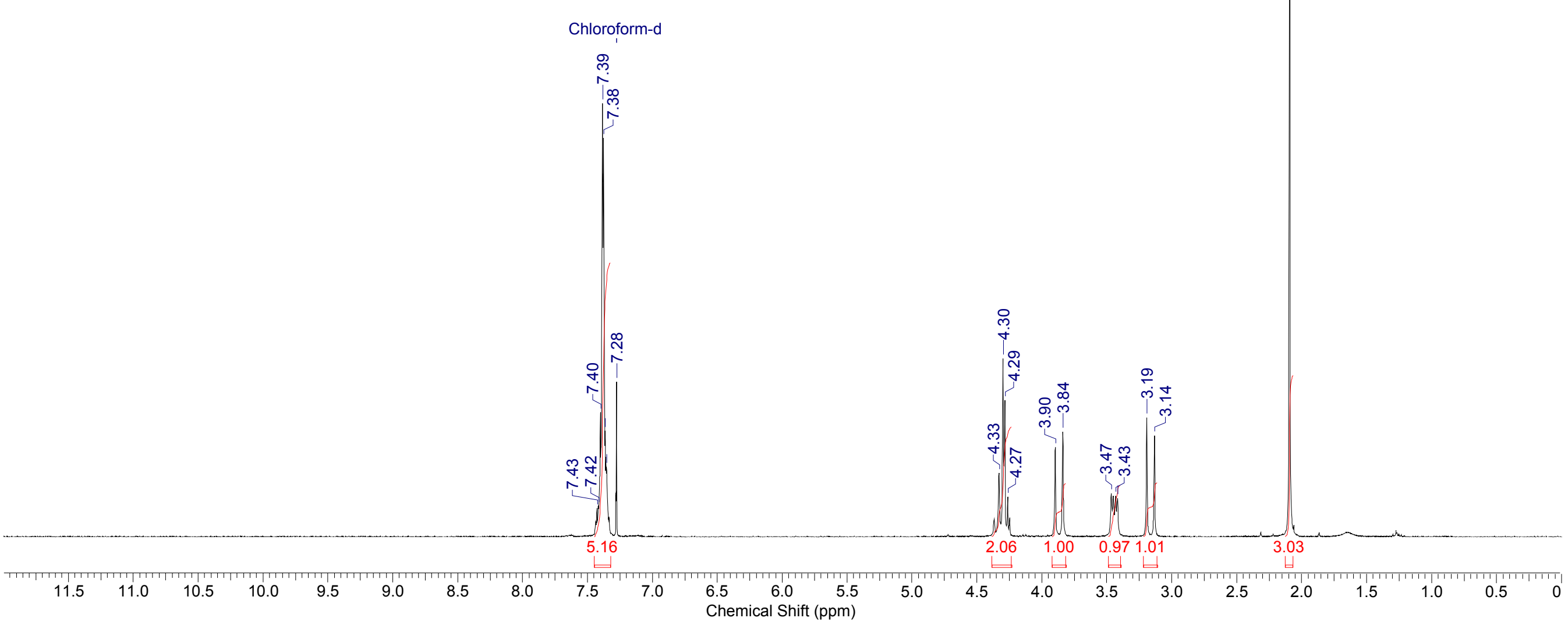




\begin{tabular}{|c|c|c|c|c|c|c|}
\hline Acquisition Time (sec) & 0.9110 & Comment & $13 \mathrm{C}$ & coupling & Date & 27 Jul 2007 19:19:00 \\
\hline File Name & \multicolumn{4}{|c|}{ C:IDocuments and SettingsIOwnerlDesktopIMAX_NMRIMax_HRO_060001r } & Frequency $(\mathrm{MHz})$ & 75.47 \\
\hline Nucleus & $13 \mathrm{C}$ & Number of Transients & \multirow{2}{*}{\multicolumn{2}{|c|}{\begin{tabular}{l|l}
127 & Original Points Count \\
CHLOROFORM-D
\end{tabular}}} & Points Count & 65536 \\
\hline Pulse Sequence & zgpg30 & Solvent & & & Sweep Width $(\mathrm{Hz})$ & 17985.61 \\
\hline
\end{tabular}

Temperature (degree C) 27.000<smiles>CN1CC(=O)OC[C@H]1c1ccccc1</smiles>

3b

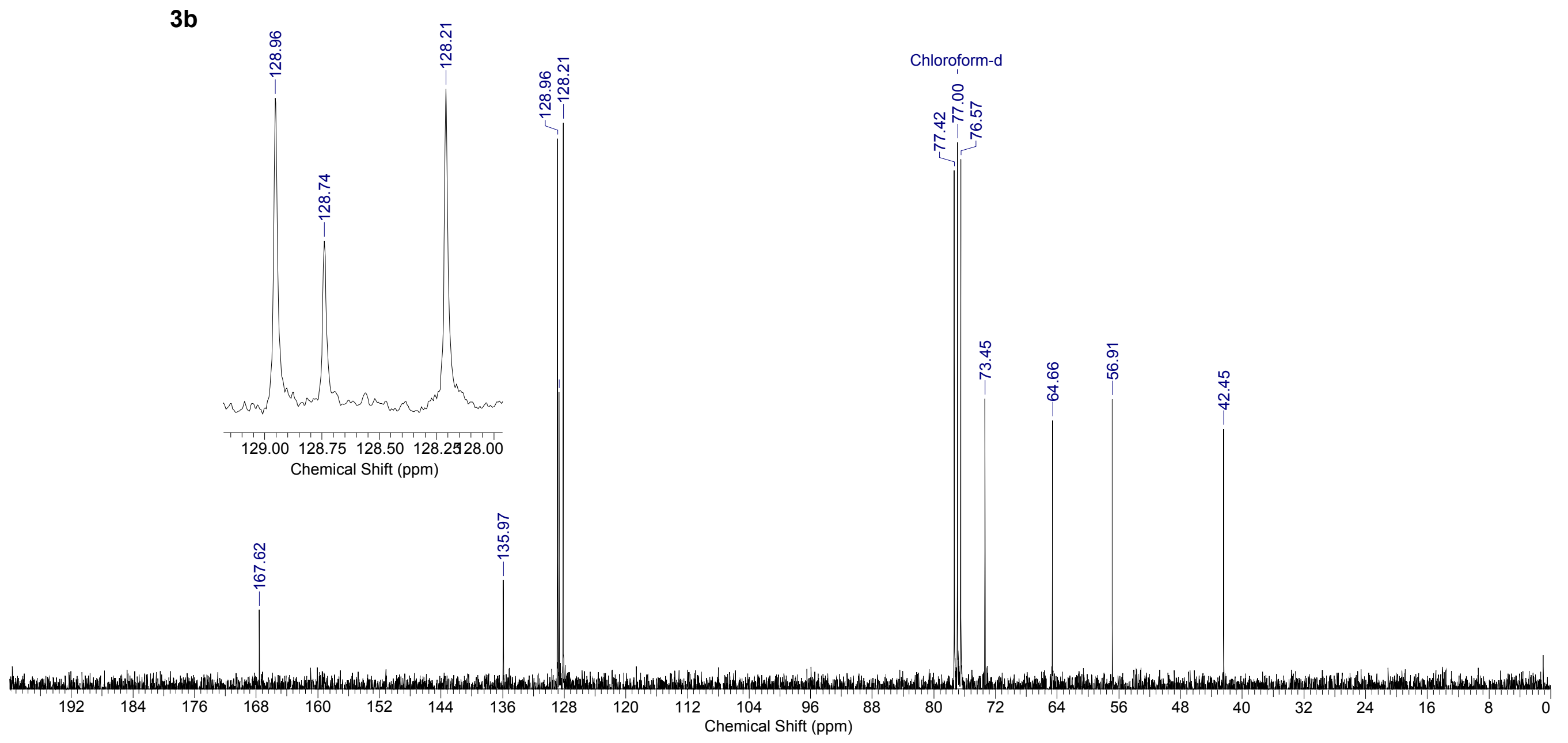


\begin{tabular}{|ll|lr}
\hline Acquisition Time (sec) & 3.0228 & Date & 18 Sep 2007 21:05:00
\end{tabular}

File Name C:IDocuments and SettingsIOwner|DesktoplMAX_NMRIMax_HRO_112001r

Nucleus $1 \mathrm{H}$

Pulse Sequence $\quad \mathrm{zg} 30$

Original Points Count 15360

Frequency $(\mathrm{MHz})$

Points Count

300.13

$\mathrm{zg} 30$ Solvent CHLOROFORM-D

Sweep Width $(\mathrm{Hz})$

Temperature (degree C) 27.000

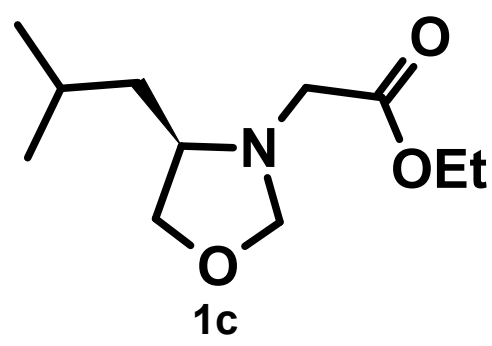

Chloroform-d
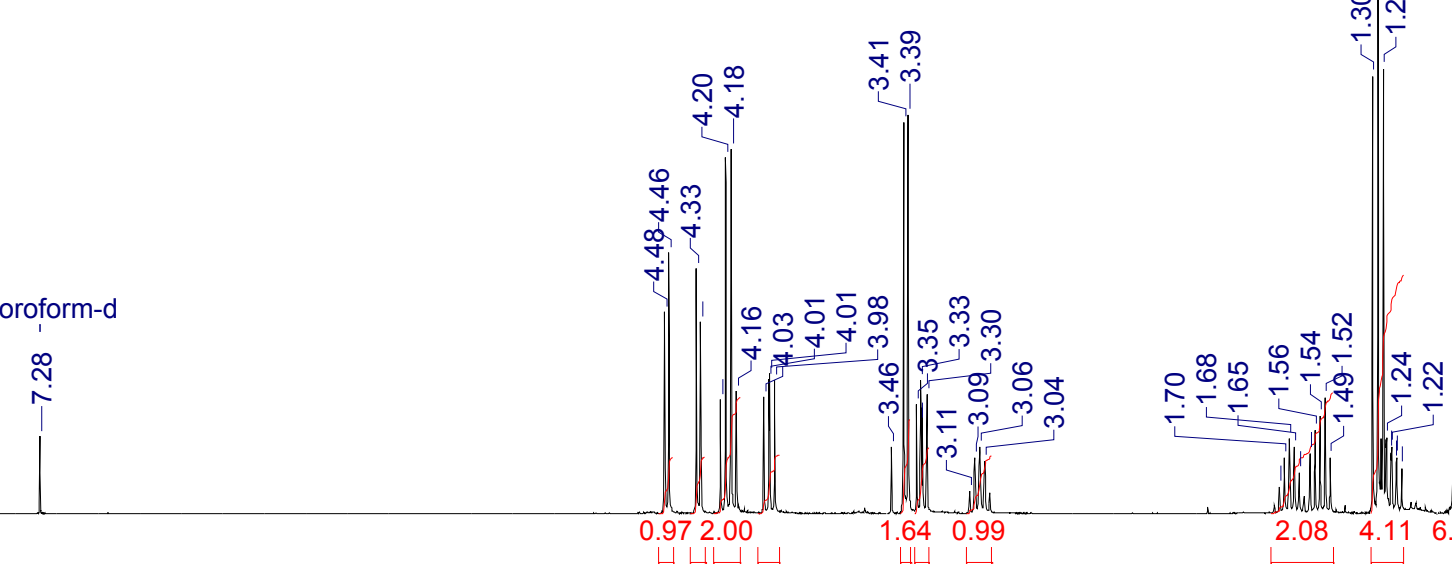


\begin{tabular}{|c|c|c|c|c|c|c|}
\hline Acquisition Time (sec) & 0.9110 & Comment & \multicolumn{2}{|c|}{$13 \mathrm{C}$ with proton decoupling } & \multirow{2}{*}{\multicolumn{2}{|c|}{$\begin{array}{ll}\text { Date } & 18 \text { Sep } 2007 \text { 21:10:00 } \\
\text { Frequency }(\mathbf{M H z}) & 75.47 \\
\end{array}$}} \\
\hline File Name & C:IDoct & Settings|Owner|Desktopln & $\mathrm{X} \_\mathrm{NI}$ & $114001 \mathrm{r}$ & & \\
\hline Nucleus & $13 \mathrm{C}$ & Number of Transients & 117 & Original Points Count & Points Count & 65536 \\
\hline Pulse Sequence & zgpg30 & Solvent & $\mathrm{CHL}$ & & Sweep Width $(\mathrm{Hz})$ & 17985.61 \\
\hline
\end{tabular}

\begin{tabular}{|ll|}
\hline Pulse Sequence & zgpg30 \\
\hline Temperature (degree C) 27.000
\end{tabular}

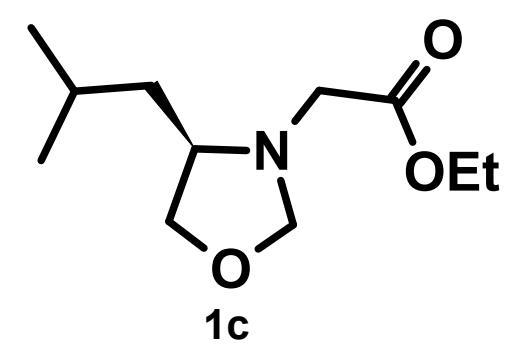

Chloroform-d
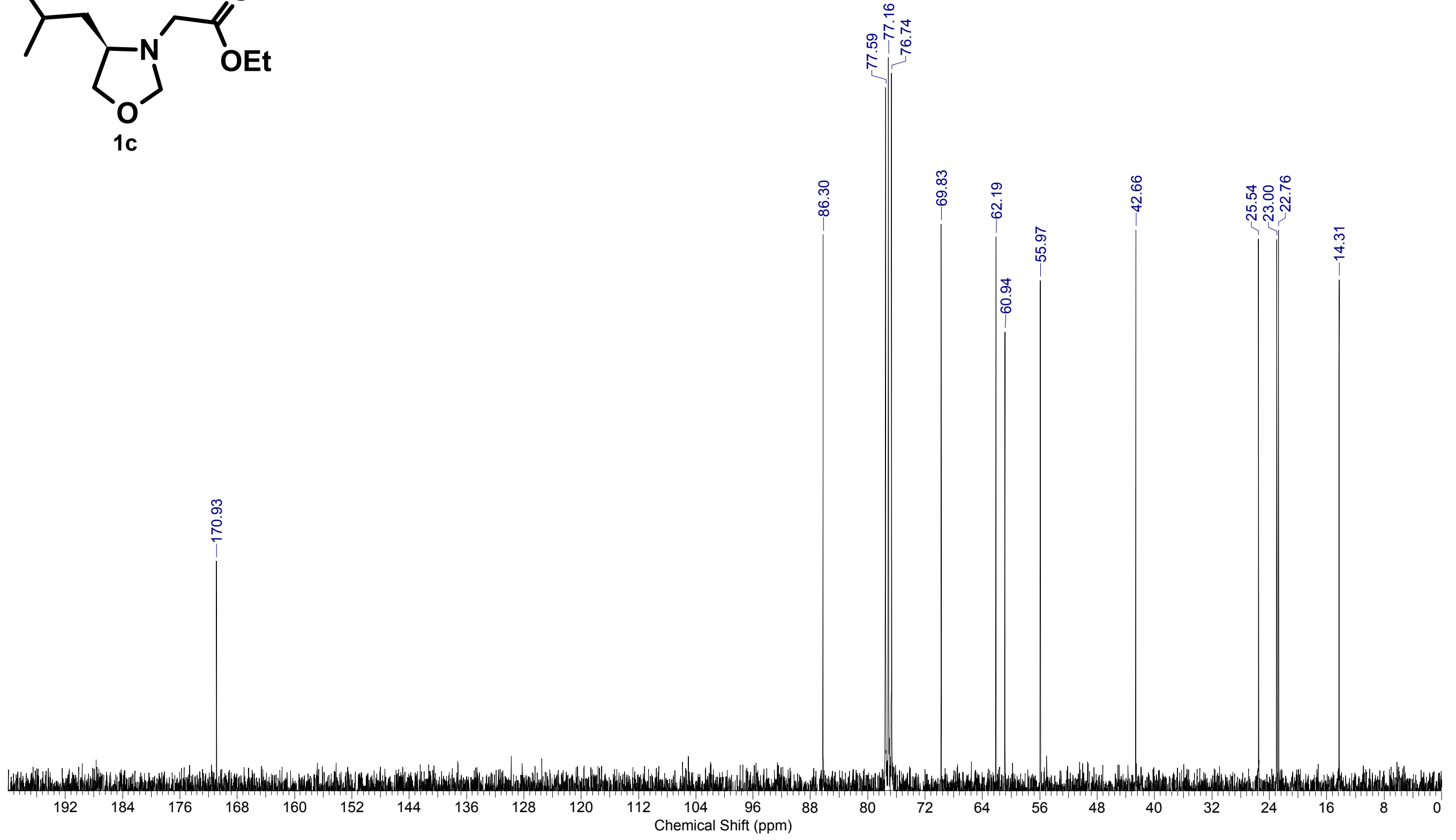
\begin{tabular}{|ll|lr}
\hline Acquisition Time (sec) & 3.0228 & Date & 26 Sep 2007 16:07:00 \\
\hline File & C: &
\end{tabular}

File Name $\quad$ C:IDocuments and SettingsiOwnerlDesktopIMAX_NMRIMax_HRO_121001r

Nucleus $\quad 1 \mathrm{H}$

Pulse Sequence $\quad \mathrm{zg} 30$

Original Points Count 15360

Frequency $(\mathrm{MHz})$

Points Count

2930

Solvent

CHLOROFORM-D

Sweep Width $(\mathrm{Hz})$

Temperature (degree C) 27.000

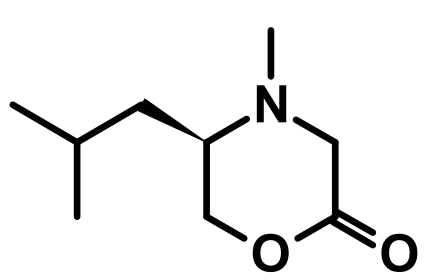

3c

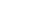

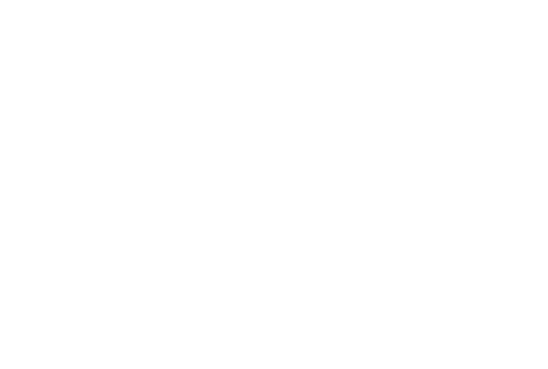

Chloroform-d

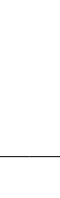

\begin{tabular}{lllllllllllllll}
\hline 11.5 & 11.0 & 10.5 & 10.0 & 9.5 & 9.0 & 8.5 & 8.0 & 7.5 & 7.0 & 6.5 & 6.0 & 5.5 \\
& & & & & & & & & & Chemical Shift (ppm)
\end{tabular}




\begin{tabular}{|c|c|c|c|c|c|c|}
\hline Acquisition Time (sec) & \multicolumn{4}{|c|}{\begin{tabular}{l|ll}
0.9110 & Comment & $13 \mathrm{C}$ with proton decoupling \\
\end{tabular}} & Date & 26 Sep 2007 16:09:00 \\
\hline File Name & \multicolumn{4}{|c|}{ C:IDocuments and SettingsIOwner|DesktopIMAX_NMRIMax_HRO_122001r } & Frequency $(\mathrm{MHz})$ & 75.47 \\
\hline Nucleus & $13 \mathrm{C}$ & Number of Transients & \multirow{2}{*}{\multicolumn{2}{|c|}{\begin{tabular}{l|l}
14 & Original Points Count \\
CHLOROFORM-D
\end{tabular}}} & Points Count & 65536 \\
\hline Pulse Sequence & zgpg30 & Solvent & & & Sweep Width $(\mathrm{Hz})$ & 17985.61 \\
\hline
\end{tabular}

Pulse Sequence zgpg30 Solvent CHLOROFORM-D

Sweep Width $(\mathrm{Hz})$

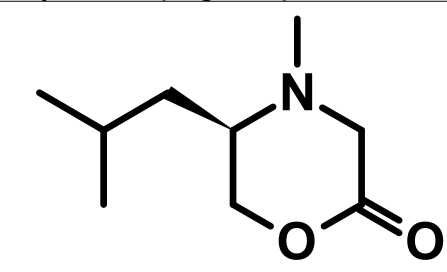

3c

Chloroform-d

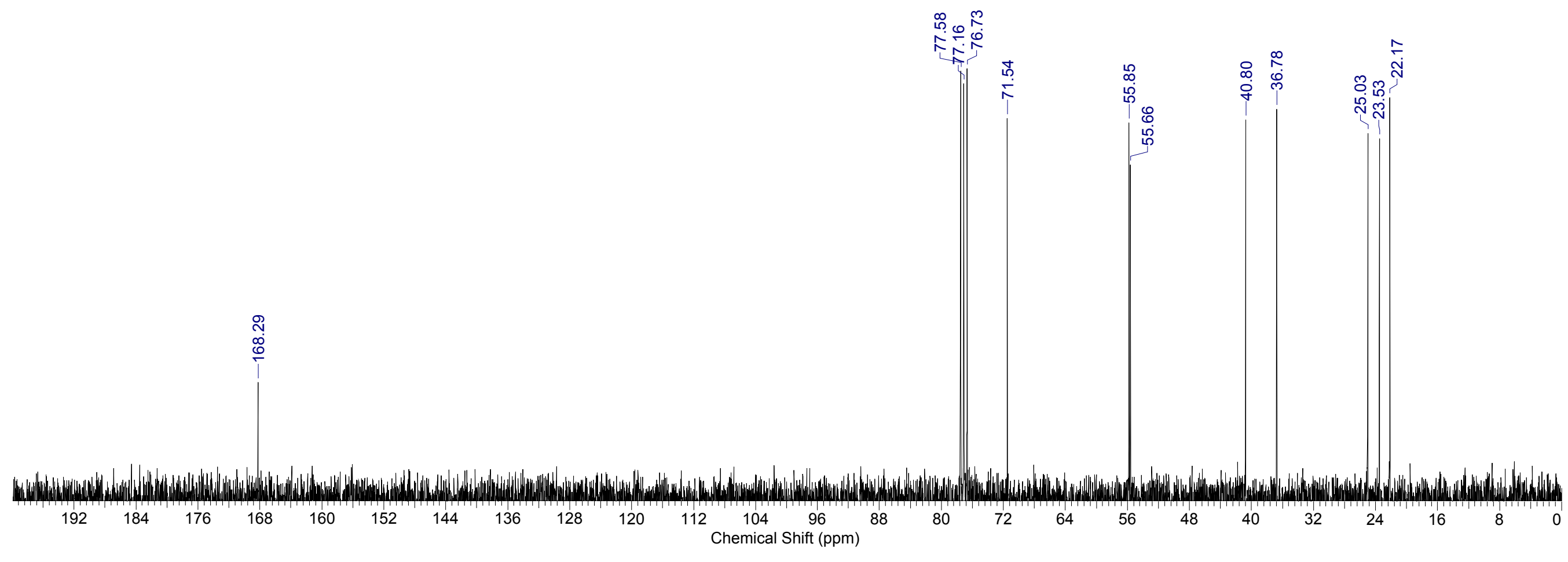


\begin{tabular}{|ll|lr}
\hline Acquisition Time (sec) & 3.0228 & Date & 09 Aug 2007 18:46:00 \\
\hline File Name & C:IDocuments and SettingsIOwnerlDesktopIMAX_NMRIMax_HRO_074
\end{tabular}

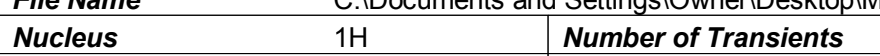

Pulse Sequence $\quad \mathrm{zg} 30$

Original Points Count 15360 Solvent

CHLOROFORM-D

Sweep Width $(\mathrm{Hz})$

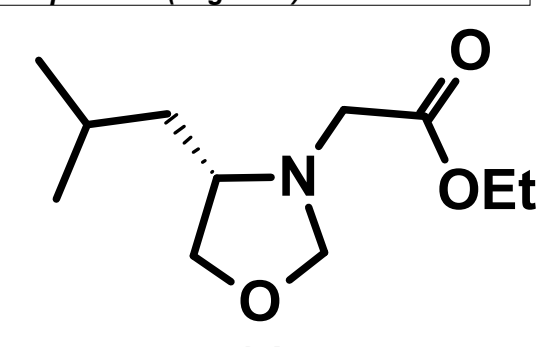

1d

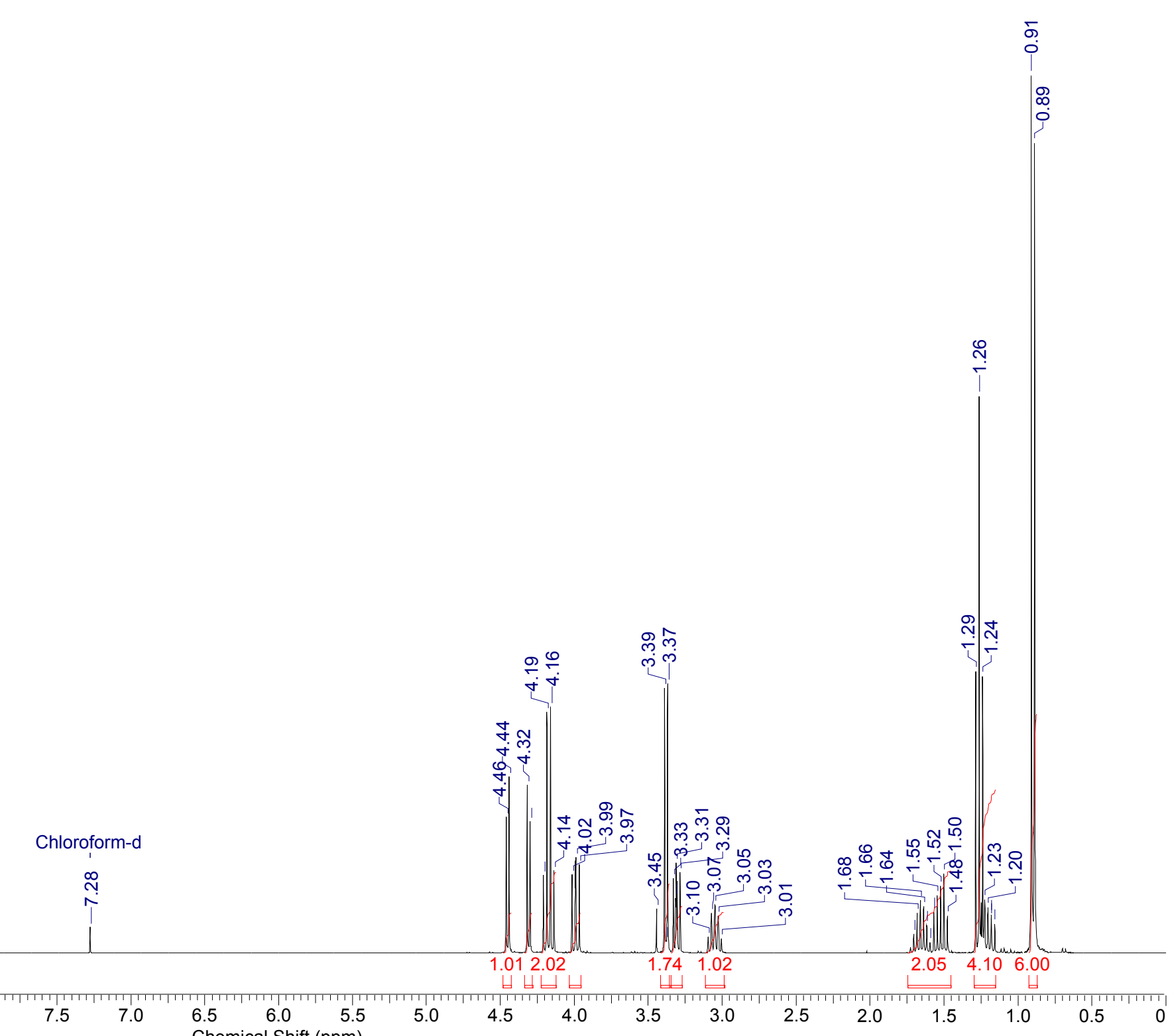




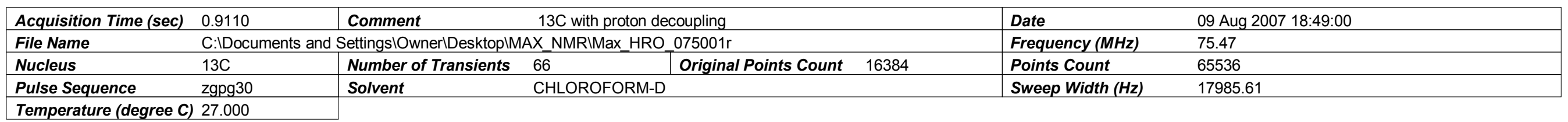
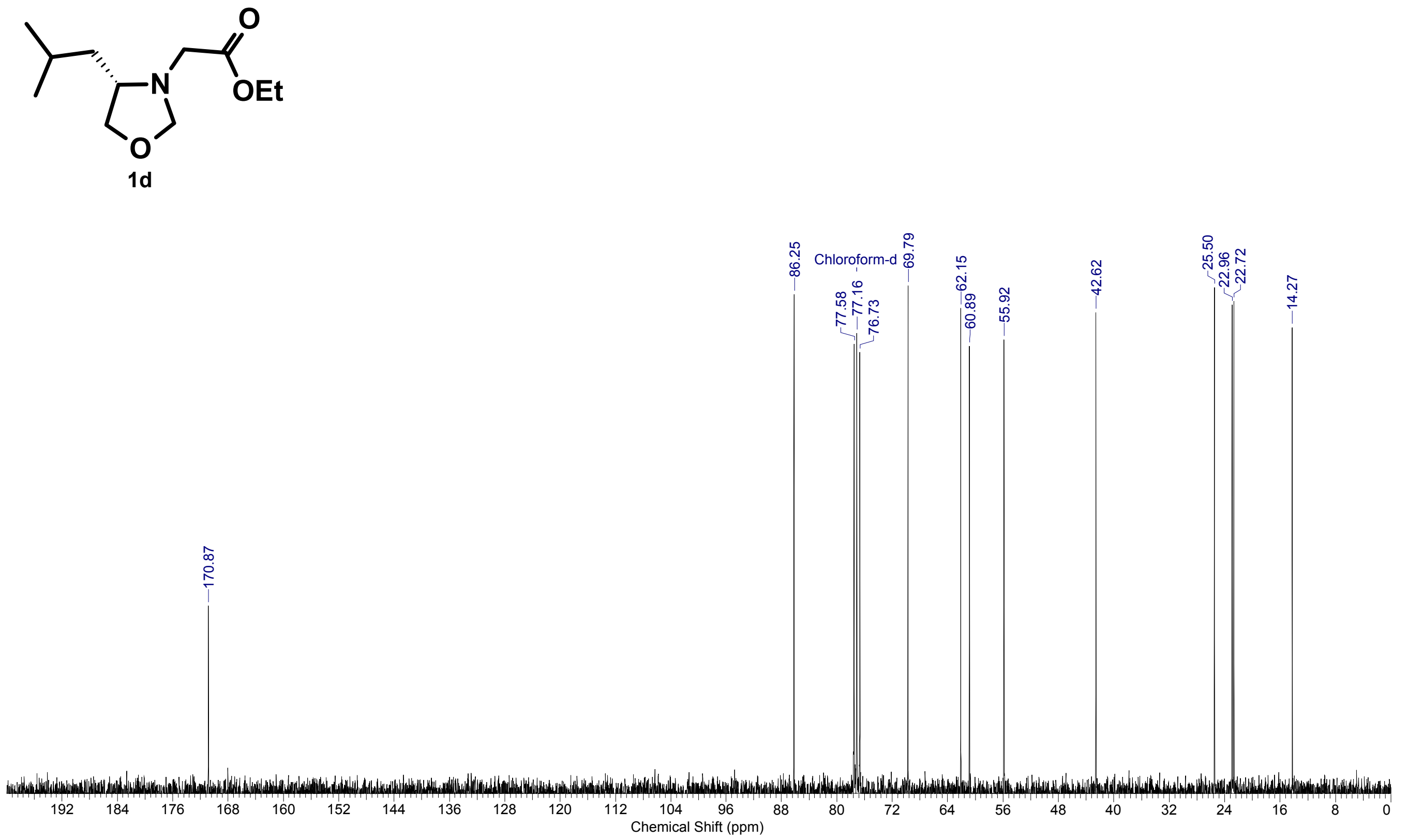
\begin{tabular}{|ll|lr|}
\hline Acquisition Time (sec) & 3.0228 & Date & 10 Aug 2007 19:14:00 \\
\hline File Name & C:IDocuments and SettingsIOwnerlDesktopIMAX_NMRIMax_HRO_076 \\
\hline Nucleus & (H)
\end{tabular}

Nucleus

Pulse Sequence $\quad \mathrm{zg} 30$

$\mathrm{zg} 30$

Solvent

CHLOROFORM-

Original Points Count 15360

Frequency $(\mathrm{MHz})$

Points Count

Temperature (degree C) 27.000

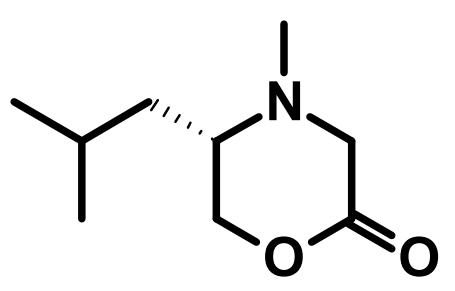

3d

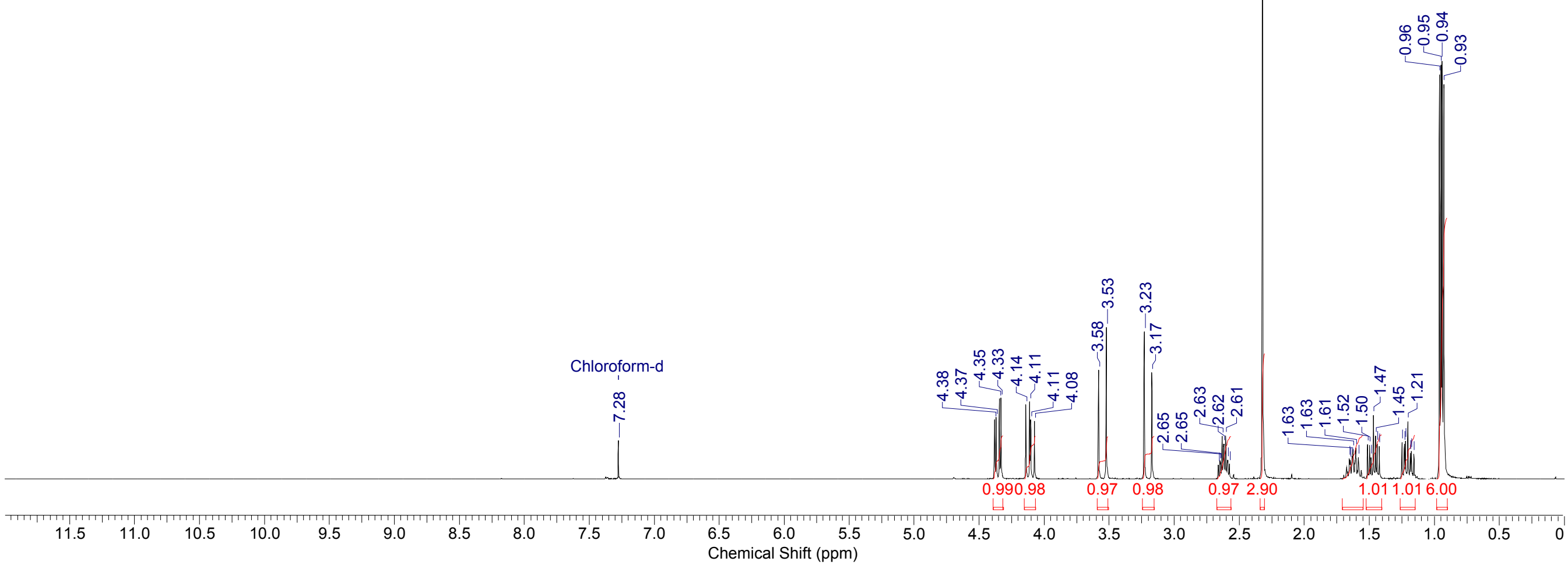




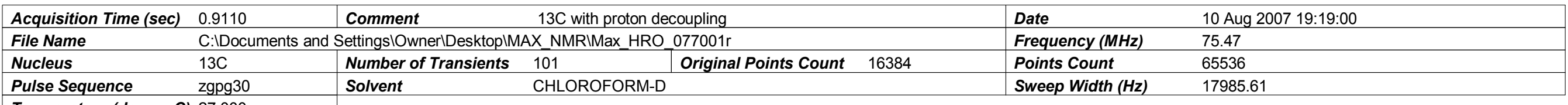

Pulse Sequence zgpg30 Solvent CHLOROFORM- 
\begin{tabular}{|ll|lr}
\hline Acquisition Time (sec) & 3.0228 & Date & 29 Aug 2007 16:05:00 \\
\hline & File &
\end{tabular}

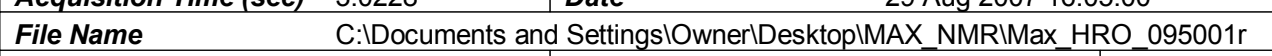

Nucleus $\quad 1 \mathrm{H} \quad$ Number of Transients

Pulse Sequence $\quad \mathrm{zg} 30$

Original Points Count 15360

Frequency $(\mathrm{MHz})$

Points Count

300.13 Solvent CHLOROFORM-D

Sweep Width $(\mathrm{Hz})$

$\sum_{0}^{\text {OEt }}$

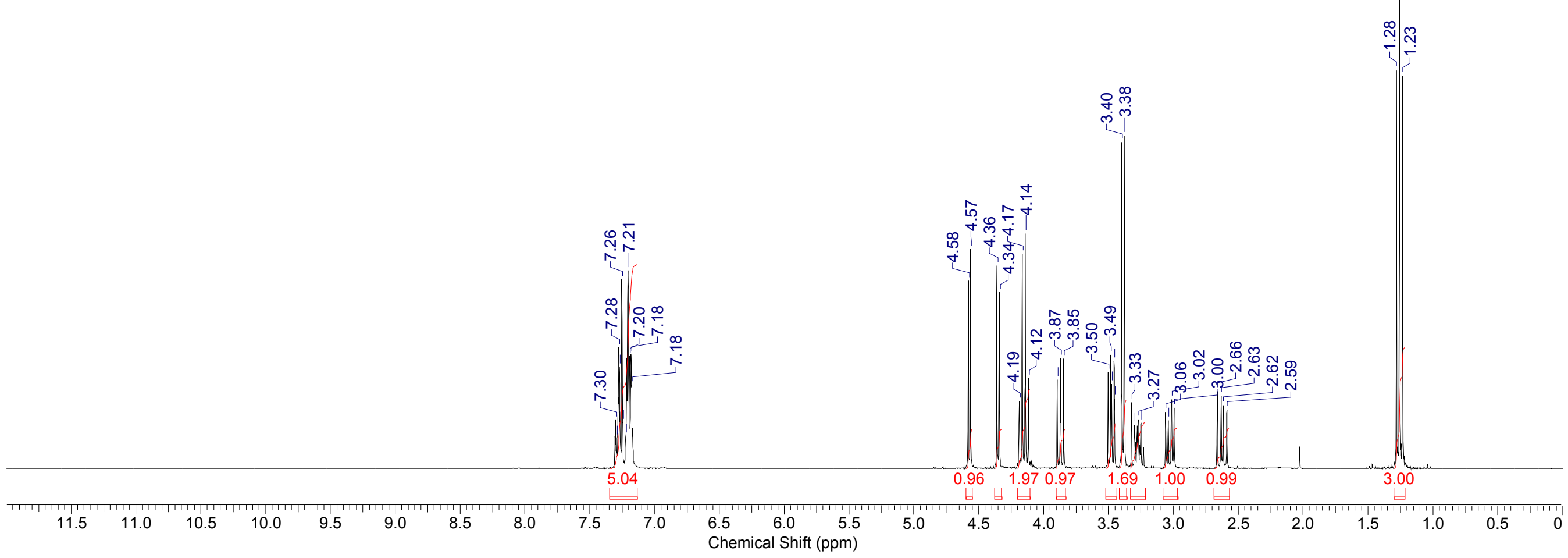




\begin{tabular}{|c|c|c|c|c|c|}
\hline Acquisition Time (sec) & 0.9110 & Comment & coupling & Date & 29 Aug 2007 16:08:00 \\
\hline File Name & \multicolumn{3}{|c|}{ C:IDocuments and Settings|Owner|DesktopIMAX_NMRIMax_HRO_096001r } & Frequency $(\mathrm{MHz})$ & 75.47 \\
\hline Nucleus & $13 \mathrm{C}$ & Number of Transients 31 & \multirow{2}{*}{\begin{tabular}{l|l}
31 & Original Points Count \\
CHLOROFORM-D & \\
\end{tabular}} & Points Count & 65536 \\
\hline Pulse Sequence & zgpg30 & Solvent & & Sweep Width $(\mathrm{Hz})$ & 17985.61 \\
\hline
\end{tabular}

Pulse Sequence zgpg30 Solvent CHLOROFORM
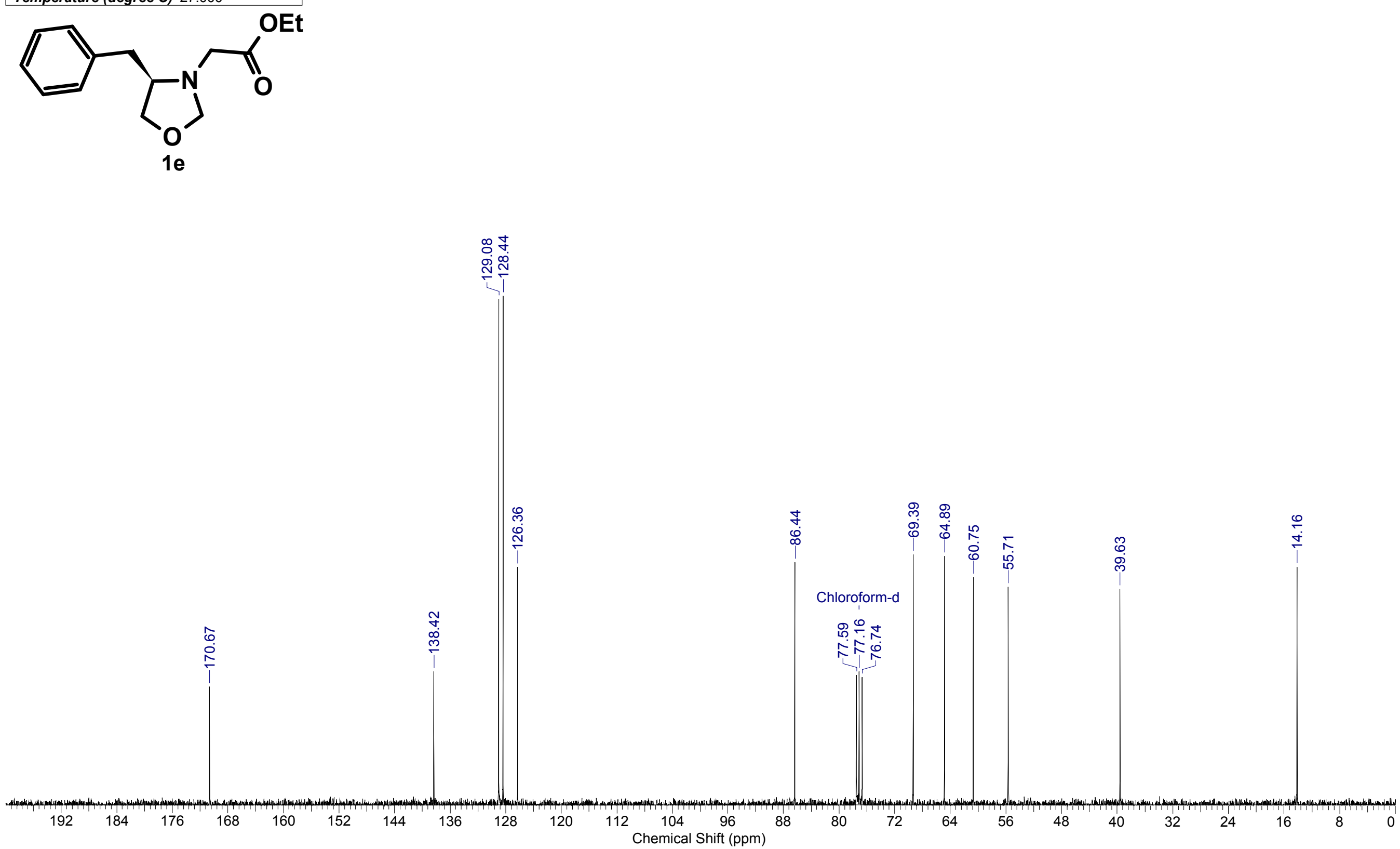
\begin{tabular}{|ll|lr}
\hline Acquisition Time (sec) & 3.0228 & Date & 31 Aug 2007 20:16:00 \\
\hline File Name & C:IDocuments and SettingsIOwnerlDesktopIMAX_NMRIMax_HRO_098
\end{tabular}

Nucleus

\begin{tabular}{ll}
\hline Pulse Sequence & $\mathrm{zg} 30$ \\
\hline Temperature (degree C) 27.000
\end{tabular}

Solvent

CHLOROFORM-D

Original Points Count 15360

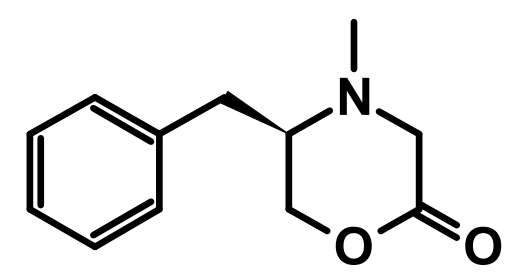

$3 e$
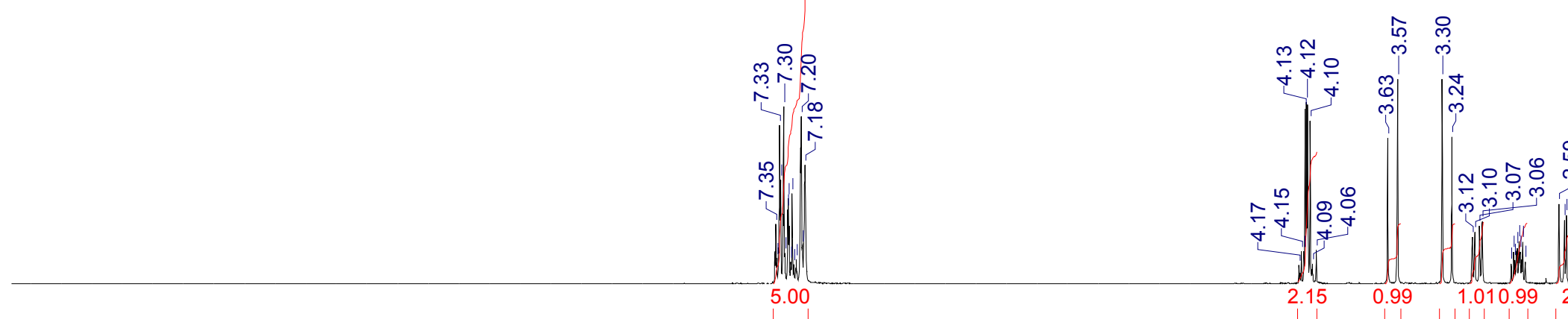

EtOAc

$\stackrel{\text { in }}{\text { in }}$ EtOAc

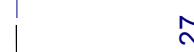

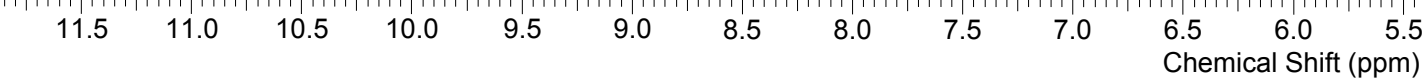




\begin{tabular}{|c|c|c|c|c|c|c|}
\hline Acquisition Time (sec) & \multicolumn{4}{|c|}{\begin{tabular}{l|ll}
0.9110 & Comment & $13 \mathrm{C}$ with proton decoupling \\
\end{tabular}} & Date & 31 Aug 2007 20:19:00 \\
\hline File Name & \multicolumn{4}{|c|}{ C:IDocuments and SettingsIOwnerlDesktopIMAX_NMRIMax_HRO_099001r } & Frequency $(\mathrm{MHz})$ & 75.47 \\
\hline Nucleus & $13 \mathrm{C}$ & Number of Transients & \multirow{2}{*}{\multicolumn{2}{|c|}{\begin{tabular}{l|l}
66 & Original Points Count \\
CHLOROFORM-D & \\
\end{tabular}}} & Points Count & 65536 \\
\hline Pulse Sequence & zgpg30 & Solvent & & & Sweep Width $(\mathrm{Hz})$ & 17985.61 \\
\hline
\end{tabular}

Pulse Sequence zgpg30 Solvent CHLOROFORM-D

Sweep Width $(\mathrm{Hz}$

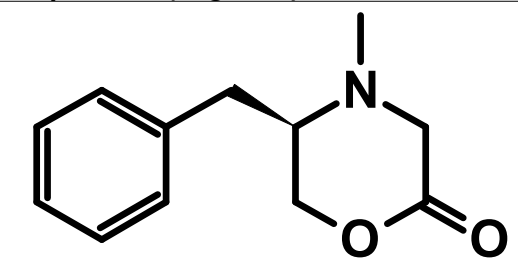

$3 e$

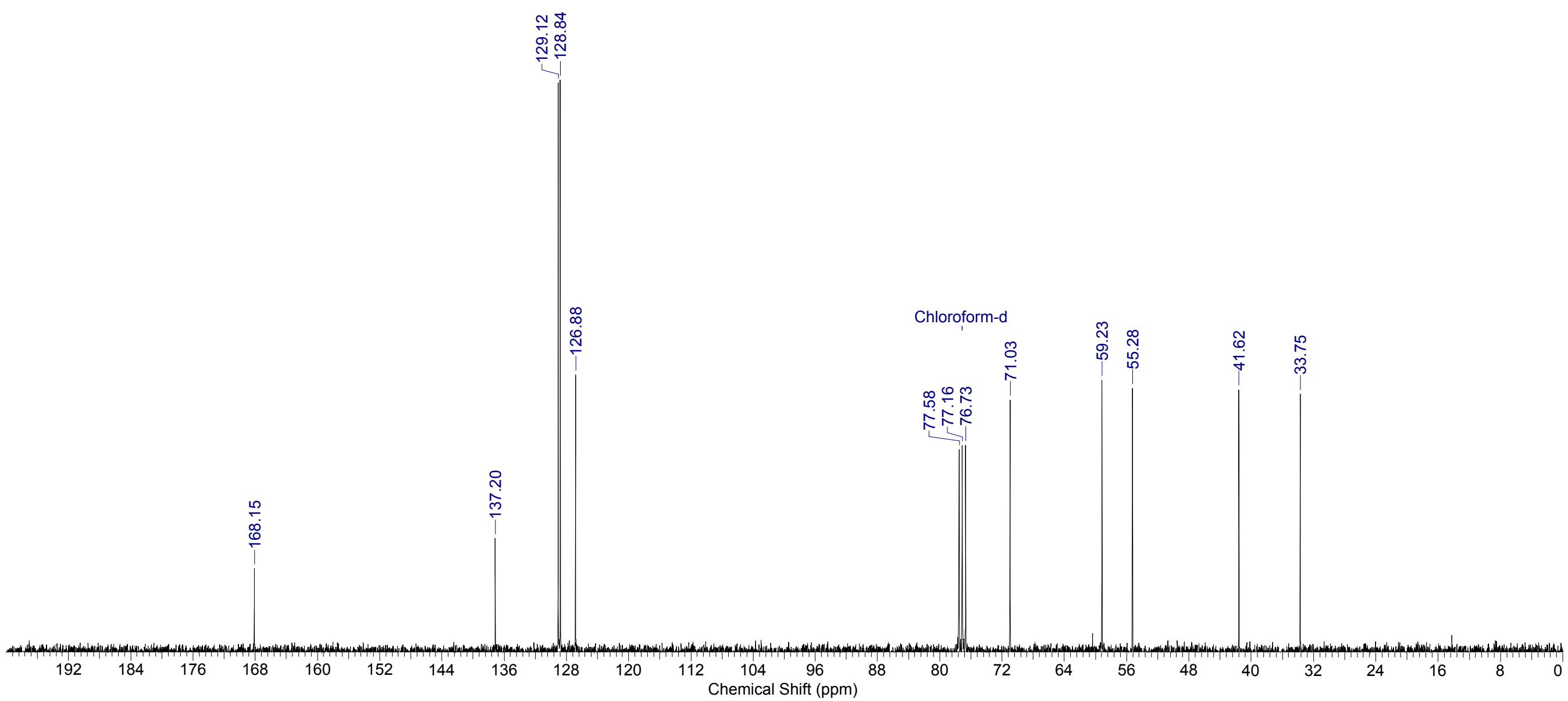


\begin{tabular}{|ll|lr}
\hline Acquisition Time (sec) & 3.0228 & Date & 22 Aug 2007 21:39:00 \\
\hline & C:Docum
\end{tabular}

File Name C:IDocuments and SettingsIOwnerlDesktopIMAX_NMRIMax_HRO_087001r

Nucleus $1 \mathrm{H}$

\begin{tabular}{|lr|}
\hline Pulse Sequence $\quad \mathrm{zg} 30$ \\
\hline Temperature (degree C) 27.000
\end{tabular}

Original Points Count 15360 Solvent CHLOROFORM-

Sweep Width $(\mathrm{Hz})$

Temperature (degree C) 27.000
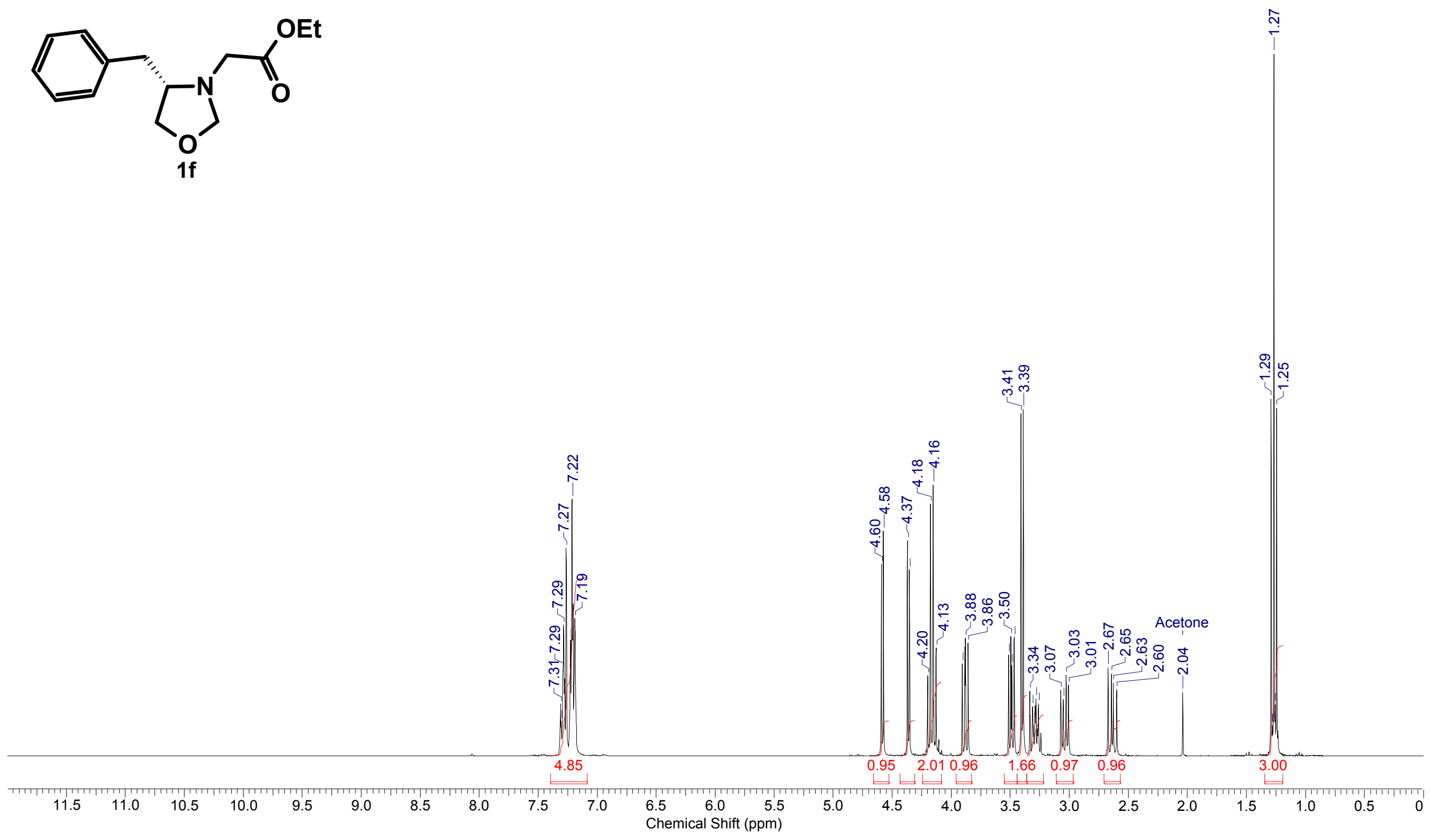


\begin{tabular}{|c|c|c|c|c|c|c|}
\hline Acquisition Time (sec) & \multicolumn{4}{|c|}{\begin{tabular}{l|ll}
0.9110 & Comment & 13C with proton decoupling \\
\end{tabular}} & Date & 22 Aug 2007 21:43:00 \\
\hline File Name & \multicolumn{4}{|c|}{ C:IDocuments and SettingslOwner|DesktoplMAX_NMRIMax_HRO_088001r } & Frequency $(\mathrm{MHz})$ & 75.47 \\
\hline Nucleus & $13 \mathrm{C}$ & Number of Transients & \multirow{2}{*}{\multicolumn{2}{|c|}{$\begin{array}{ll}68 & \text { Original Points Count } \\
\text { CHLOROFORM-D }\end{array}$}} & Points Count & 65536 \\
\hline Pulse Sequence & zgpg30 & Solvent & & & Sweep Width $(\mathrm{Hz})$ & 17985.61 \\
\hline
\end{tabular}

Pulse Sequence zgpg30 Solvent CHLOROFORM-D

Sweep Width $(\mathrm{Hz})$
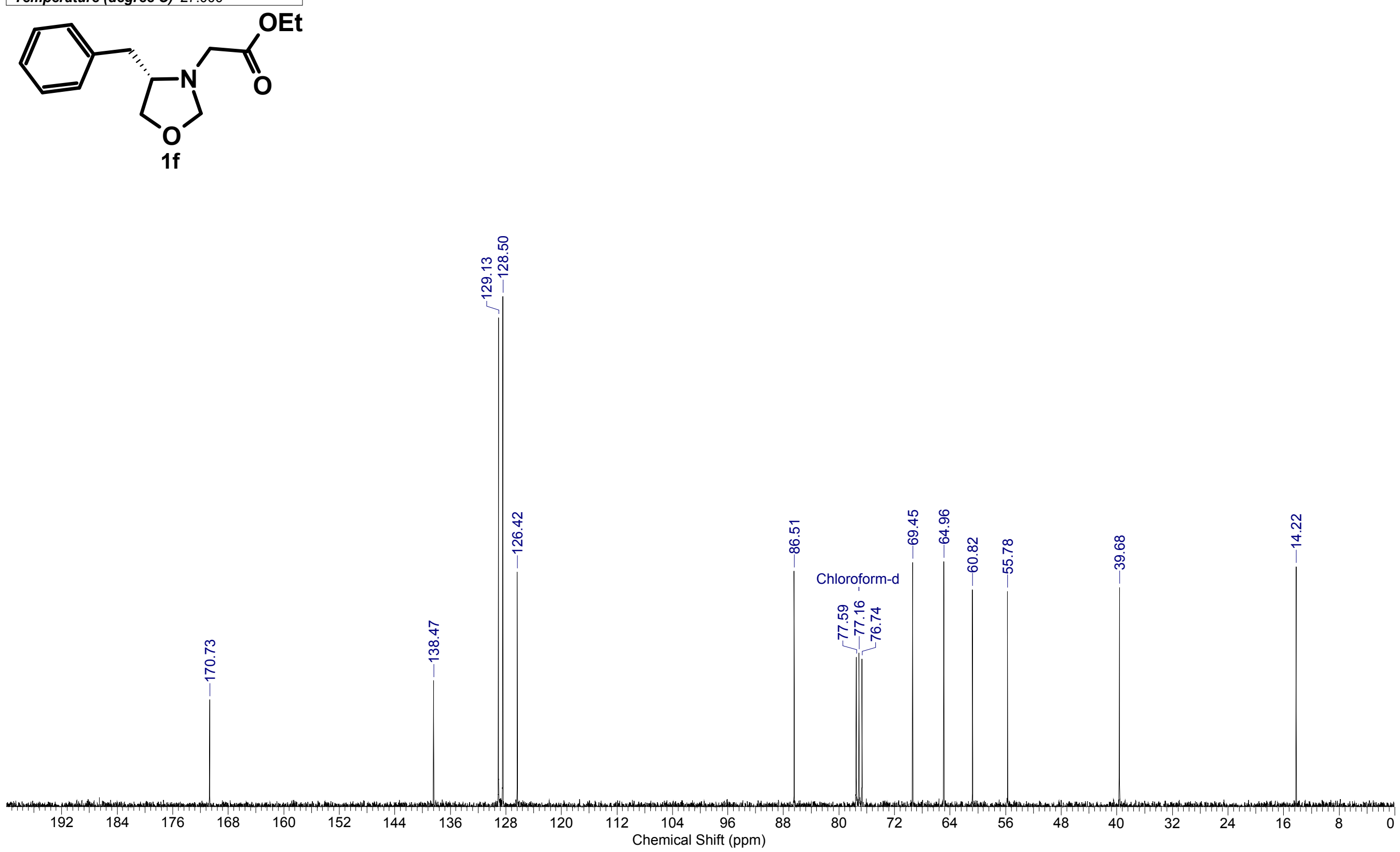


\begin{tabular}{|c|c|c|c|c|c|c|c|}
\hline \multirow{2}{*}{$\begin{array}{l}\text { Acquisition Time (sec) } \\
\text { File Name }\end{array}$} & \multirow{2}{*}{\multicolumn{5}{|c|}{\begin{tabular}{l|lc|c}
0.9110 & Comment & 13C DEPT 135 & Date \\
C:IDocuments and SettingslOwner|DesktopIMAX NMRIMax HRO $089001 \mathrm{r}$
\end{tabular}}} & \multirow{3}{*}{\begin{tabular}{|l|} 
Frequency (MHz) \\
Points Count \\
\end{tabular}} & \multirow{3}{*}{\begin{tabular}{|l}
75.47 \\
65536 \\
\end{tabular}} \\
\hline & & & & & & & \\
\hline Nucleus & $13 \mathrm{C}$ & Number of Transients & 66 & Original Points Coun & 16384 & & \\
\hline Pulse Sequence & dept135 & Solvent & CHLOROFORM & & & Sweep Width $(\mathrm{Hz})$ & 17985.61 \\
\hline
\end{tabular}

Temperature (degree C) 27.000

$\stackrel{2}{\check{2}}$

$\Delta$<smiles>CCOC(=O)CN1CO[C@H](C)C1</smiles>

$\stackrel{\leftarrow}{\stackrel{\leftarrow}{*}}$

$\stackrel{m}{\mathfrak{0}}$

$\stackrel{8}{\circ}$

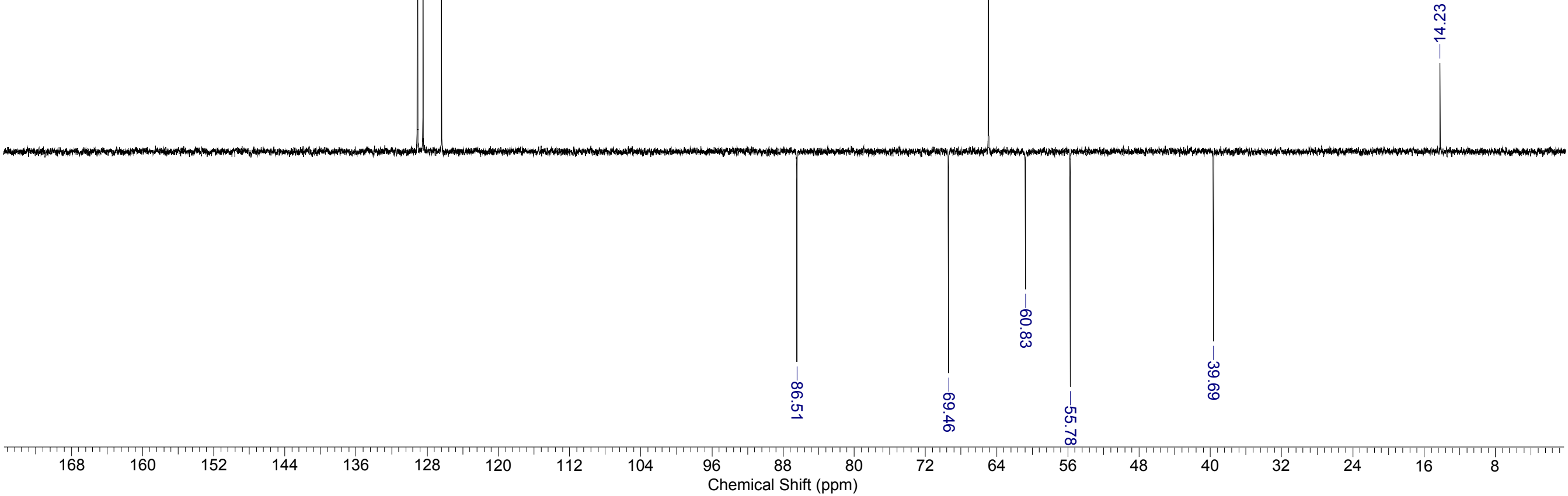


\begin{tabular}{|ll|lr}
\hline Acquisition Time (sec) & 3.0228 & Date & 24 Aug 2007 20:35:00 \\
\hline File & C. &
\end{tabular}

File Name $\quad$ C:IDocuments and SettingsiOwnerlDesktopIMAX_NMRIMax_HRO_092001r

\begin{tabular}{ll|l} 
Nucleus & $1 \mathrm{H}$ & Number of Transients 4
\end{tabular}

Pulse Sequence $\quad \mathrm{zg} 30$

Original Points Count 15360

Frequency $(\mathrm{MHz})$

Points Count

300.13 Solvent CHLOROFORM-D

Sweep Width $(\mathrm{Hz})$

$P_{0}$

$3 f$

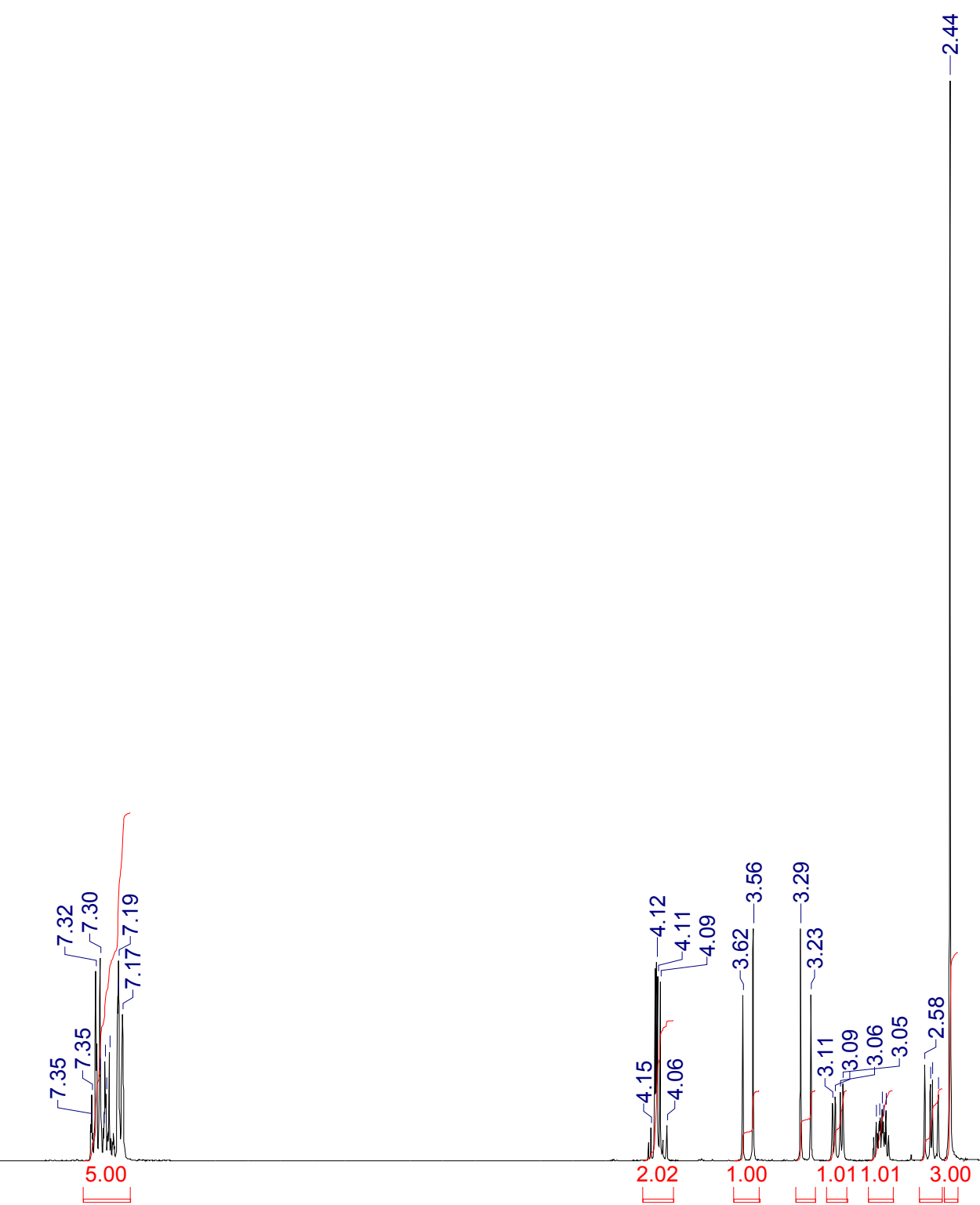

$\begin{array}{llllllllllllll}11.5 & 11.0 & 10.5 & 10.0 & 9.5 & 9.0 & 8.5 & 8.0 & 7.5 & 7.0 & 6.5 & 6.0 & 5.5 \\ & & & & & & & & & & \text { Chemical Shift (ppm) }\end{array}$

$5.0 \quad 4.5$

4.01035

$+\frac{1}{3.0}$

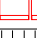




\begin{tabular}{|c|c|c|c|c|c|c|}
\hline Acquisition Time (sec) & \multicolumn{4}{|c|}{\begin{tabular}{l|ll}
0.9110 & Comment & $13 \mathrm{C}$ with proton decoupling \\
\end{tabular}} & Date & 24 Aug 2007 20:37:00 \\
\hline File Name & \multicolumn{4}{|c|}{ C:IDocuments and SettingslOwner|DesktoplMAX_NMRIMax_HRO_093001r } & Frequency $(\mathrm{MHz})$ & 75.47 \\
\hline Nucleus & $13 \mathrm{C}$ & Number of Transients & \multirow{2}{*}{\multicolumn{2}{|c|}{$\begin{array}{ll}48 & \text { Original Points Count } \\
\text { CHLOROFORM-D }\end{array}$}} & Points Count & 65536 \\
\hline Pulse Sequence & zgpg30 & Solvent & & & Sweep Width $(\mathrm{Hz})$ & 17985.61 \\
\hline
\end{tabular}

Pulse Sequence zgpg30 Solvent CHLOROFORM-D

Sweep Width $(\mathrm{Hz}$

$\sigma r_{0} \lambda_{0}$

3f

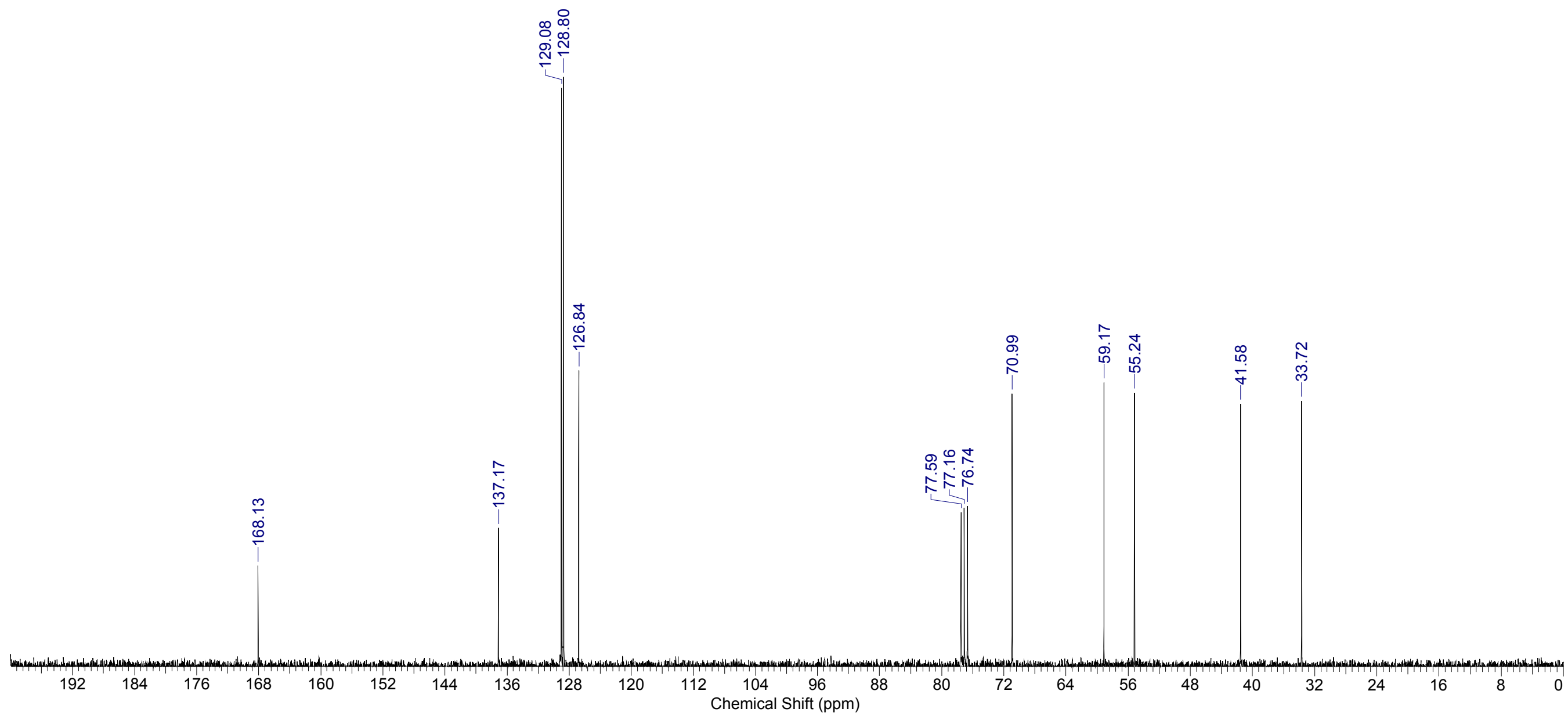




\begin{tabular}{|c|c|c|c|c|c|c|c|}
\hline Acquisition Time (sec) & 0.9110 & Comment & 13C DEPT 135 & Date & \multicolumn{3}{|c|}{24 Aug 2007 20:39:00 } \\
\hline File Name & \multicolumn{5}{|c|}{ C:IDocuments and Settings|Owner|DesktoplMAX_NMRIMax_HRO_094001r } & Frequency $(\mathrm{MHz})$ & 75.47 \\
\hline Nucleus & $13 \mathrm{C}$ & Number of Transients & 30 & Original Points Coun & 16384 & Points Count & 65536 \\
\hline Pulse Sequence & dept135 & Solvent & CHLOROFORM & & & Sweep Width $(\mathrm{Hz})$ & 17985.61 \\
\hline
\end{tabular}

Temperature (degree C) 27.000<smiles>[GeH2][TeH]</smiles>
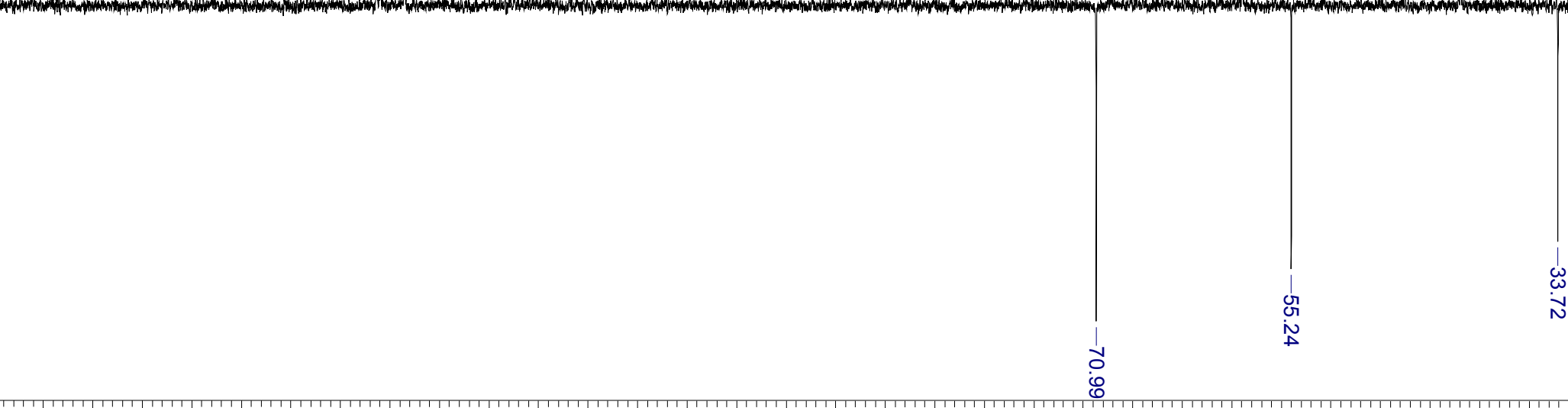
\begin{tabular}{|ll|lr}
\hline Acquisition Time (sec) & 3.0228 & Date & 01 Aug 2007 22:06:00
\end{tabular}

File Name $\quad$ C:IDocuments and SettingsiOwnerlDesktopIMAX_NMRIMax_HRO_063001r

Number of Transients 16

riginal Points Count 15360

Frequency $(\mathrm{MHz})$

Points Count

300.13

\begin{tabular}{ll} 
Nucleus & $1 \mathrm{H}$ \\
\hline Pulse Sequence & $\mathrm{zg} 30$
\end{tabular} Solvent

CHLOROFORM-D

Sweep Width $(\mathrm{Hz})$

\begin{tabular}{ll} 
Pulse Sequence & $\mathrm{zg} 30$ \\
\hline Temperature (degree C) 27.000
\end{tabular}

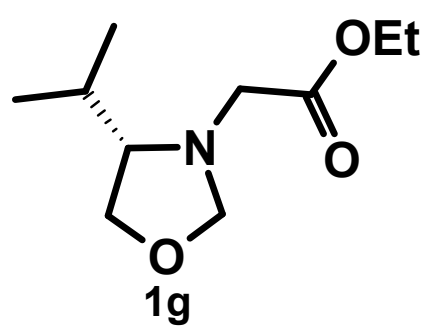

$\stackrel{m}{+}$

Chloroform-d

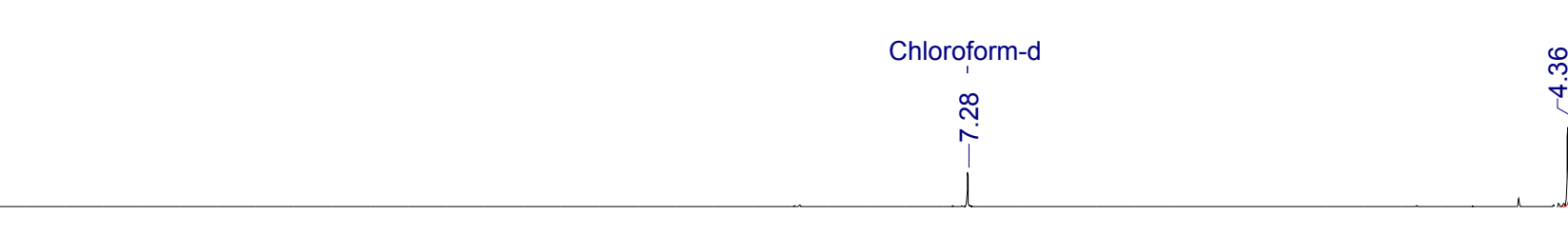

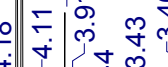

$\forall$ वल

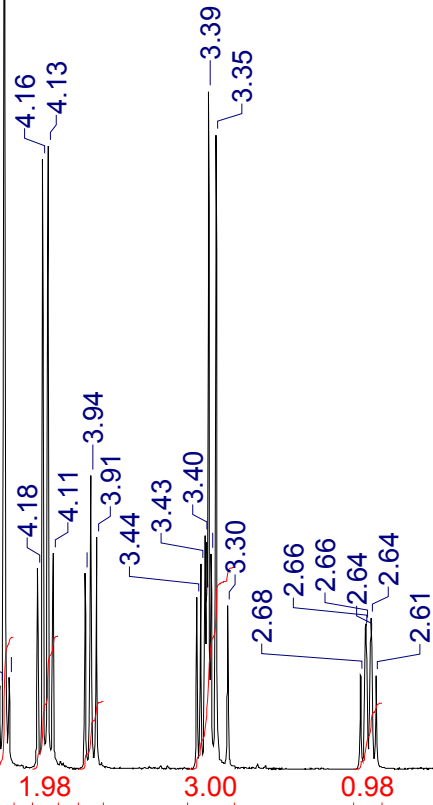




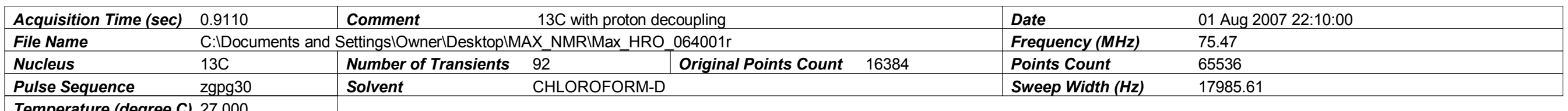

\begin{tabular}{|ll|}
\hline Pulse Sequence & zgpg30 \\
\hline Temperature (degree C) 27.000
\end{tabular}
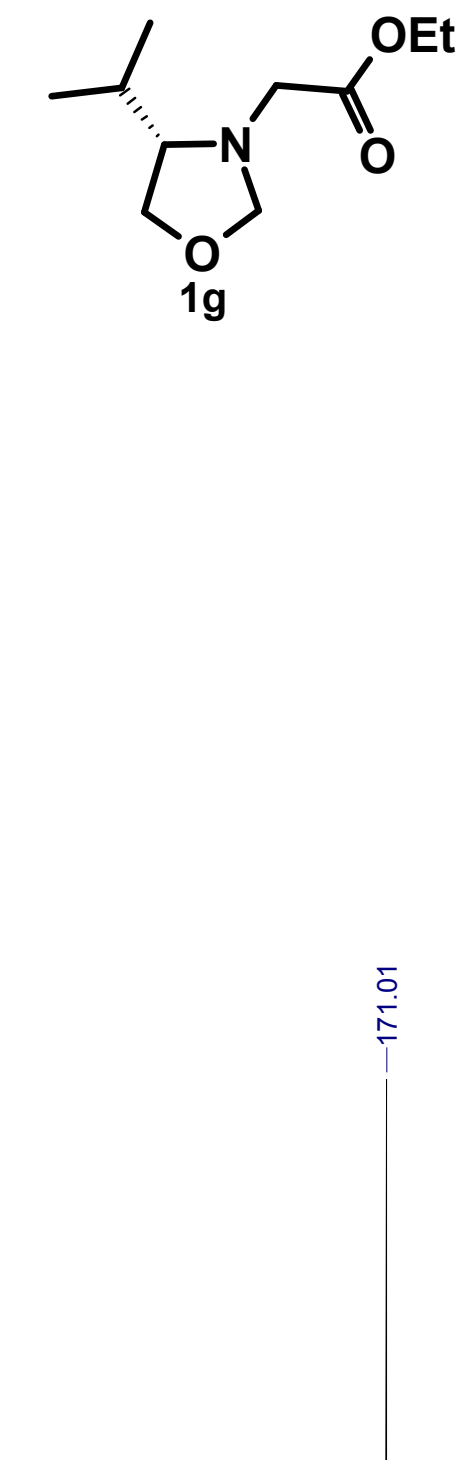

$\frac{5}{5}$

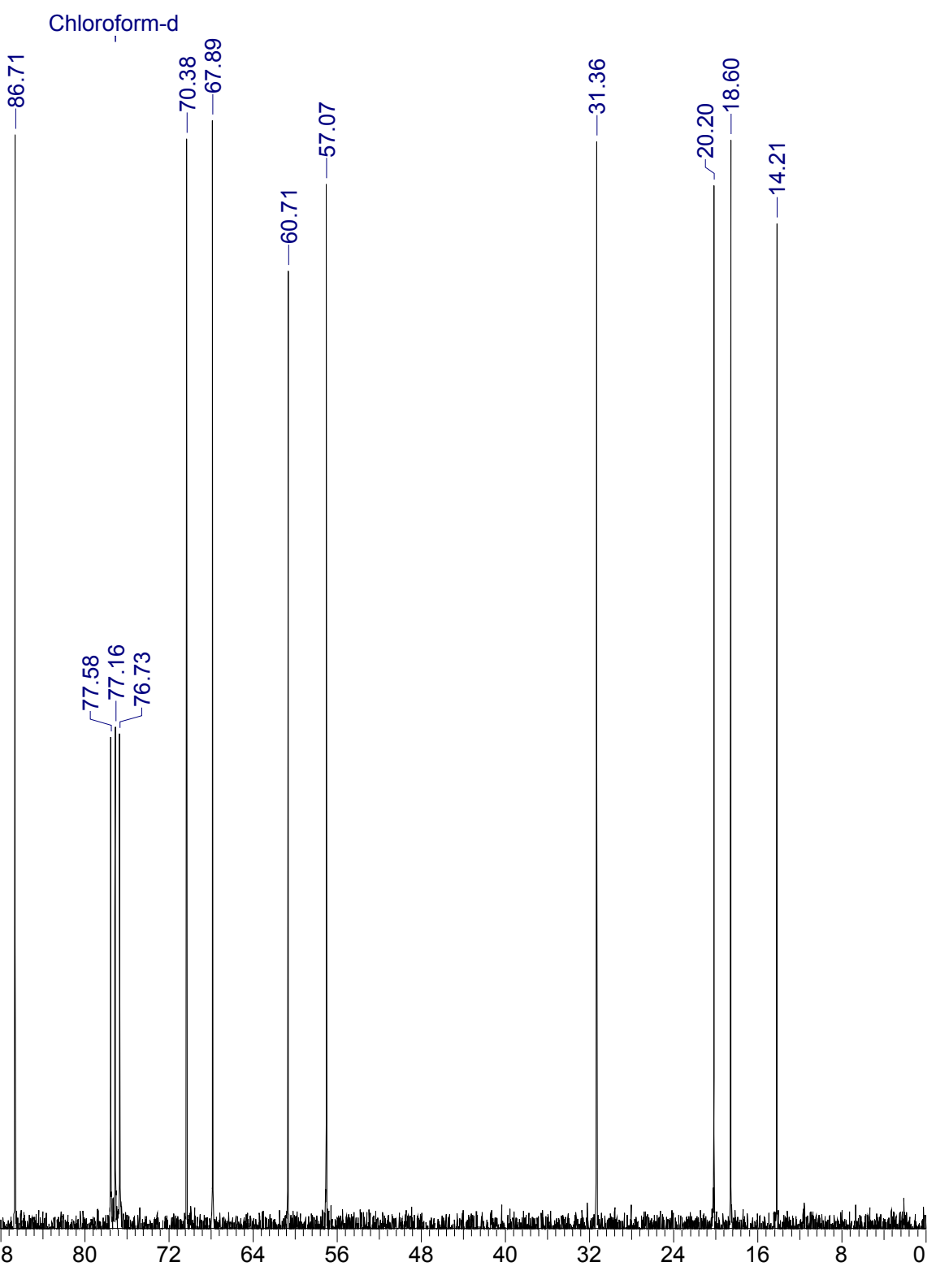


\begin{tabular}{|ll|ll|}
\hline Acquisition Time (sec) & 3.0228 & Date & 02 Aug 2007 18:44:00 \\
\hline File Name & C:IDocuments and SettingsIOwnerlDesktopIMAX_NMRIMax_HRO_06 \\
\hline Nucleus & (H) &
\end{tabular}

Nucleus \begin{tabular}{|ll|}
\hline Pulse Sequence $\quad \mathrm{zg} 30$ \\
\hline Temperature (degree C) 27.000
\end{tabular} Solvent CHLOROFORM-D

Original Points Count 15360 Solvent

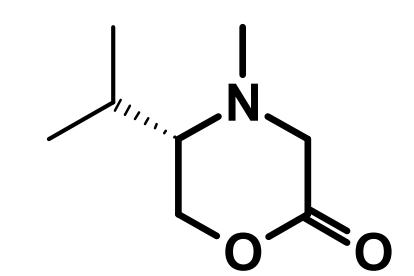

$3 g$

Sweep Width $(\mathrm{Hz})$

Chloroform-d

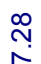




\begin{tabular}{|c|c|c|c|c|c|c|c|}
\hline \multirow{4}{*}{$\begin{array}{l}\text { Acquisition Time (sec) } \\
\text { File Name } \\
\text { Nucleus } \\
\text { Pulse Sequence }\end{array}$} & \multirow{2}{*}{\multicolumn{5}{|c|}{\begin{tabular}{l|lcl}
0.9110 & Comment & $13 \mathrm{C}$ with proton decoupling \\
$\mathrm{C}:$ IDocuments and Settings $\mid$ OwnerIDesktopIMAX_NMRIMax_HRO_067001r
\end{tabular}}} & \multirow{3}{*}{\multicolumn{2}{|c|}{$\begin{array}{ll}\text { Date } & 02 \text { Aug } 2007 \text { 18:49:00 } \\
\text { Frequency (MHz) } & 75.47 \\
\text { Points Count } & 65536 \\
\end{array}$}} \\
\hline & & & & & & & \\
\hline & $13 \mathrm{C}$ & Number of Transients & 101 & Original Points Count & 16384 & & \\
\hline & zgpg30 & Solvent & DMSO-D6 & Sweep Width $(\mathrm{Hz})$ & 17985.61 & Temperature (degree $\mathrm{C}$ ) & 27.000 \\
\hline
\end{tabular}

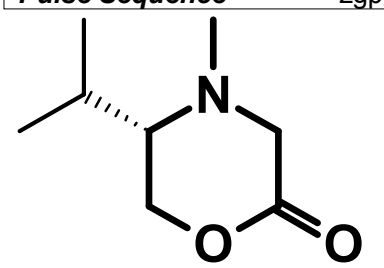

$3 g$

Chloroform-d

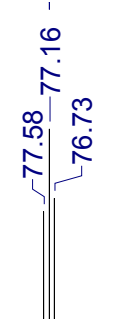

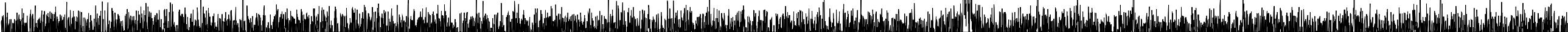
$\begin{array}{cc}104 & 96 \\ \text { Chemical Shift (ppm) }\end{array}$ 
\begin{tabular}{|ll|lr}
\hline Acquisition Time (sec) & 3.0228 & Date & 23 Oct 2007 19:03:00
\end{tabular}

File Name C:IDocuments and SettingslOwnerlDesktopIMAX_NMRIMax_HRO_145001r $1 \mathrm{H}$

\begin{tabular}{|ll|ll|}
\hline Pulse Sequence & zg30 & Solvent & CHLOROFORM-D \\
\hline
\end{tabular}

Original Points Count 15360

Frequency $(\mathrm{MHz})$

Points Count

Temperature (degree C) 27.000
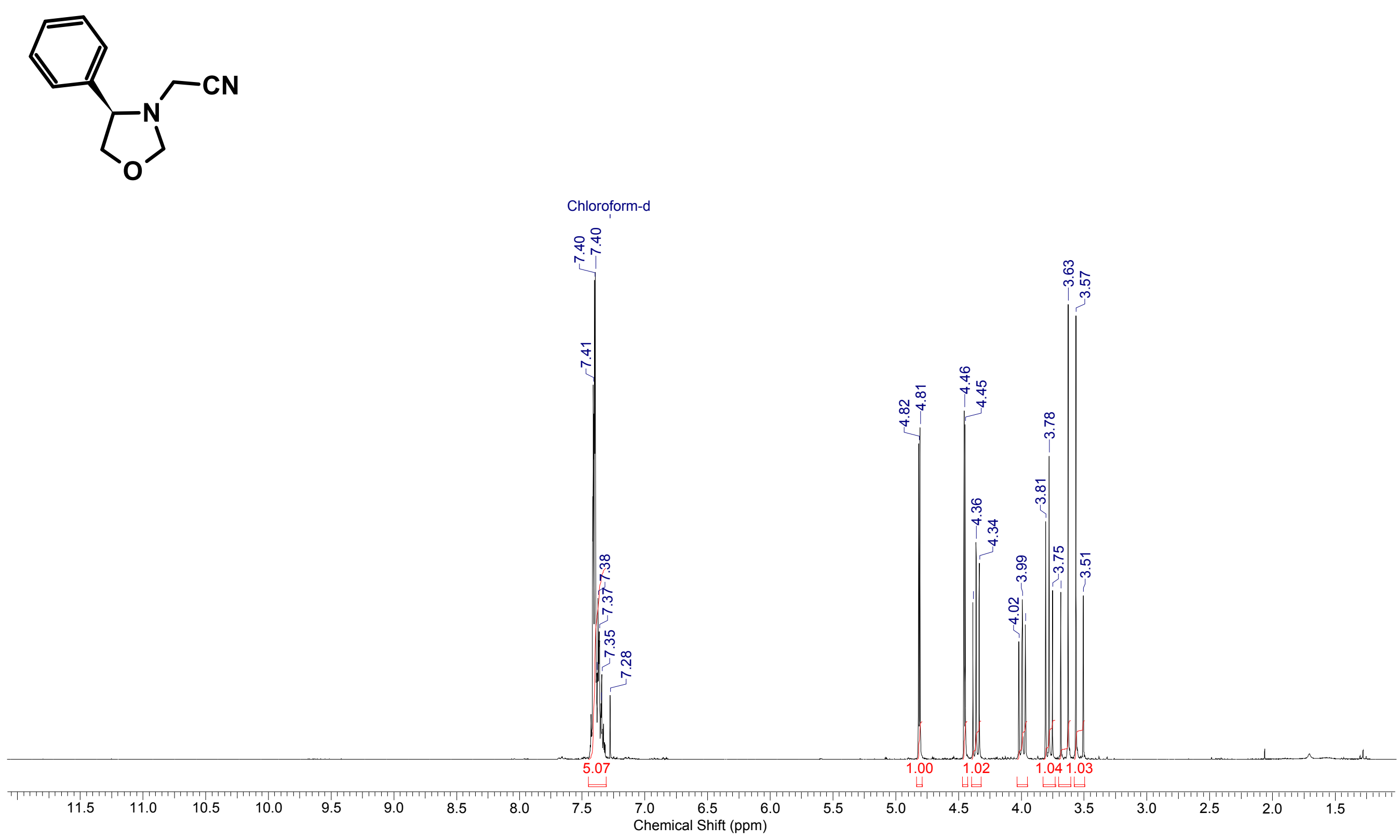


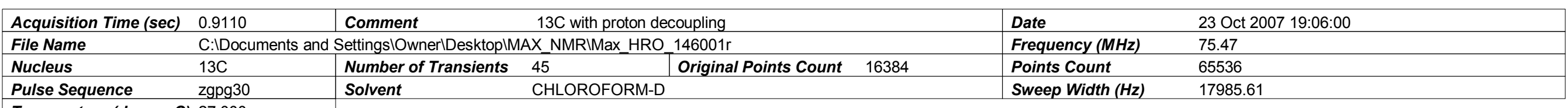

Pulse Sequence zgpg30 Solvent CHLOROFORM-

Sweep Width $(\mathrm{Hz})$
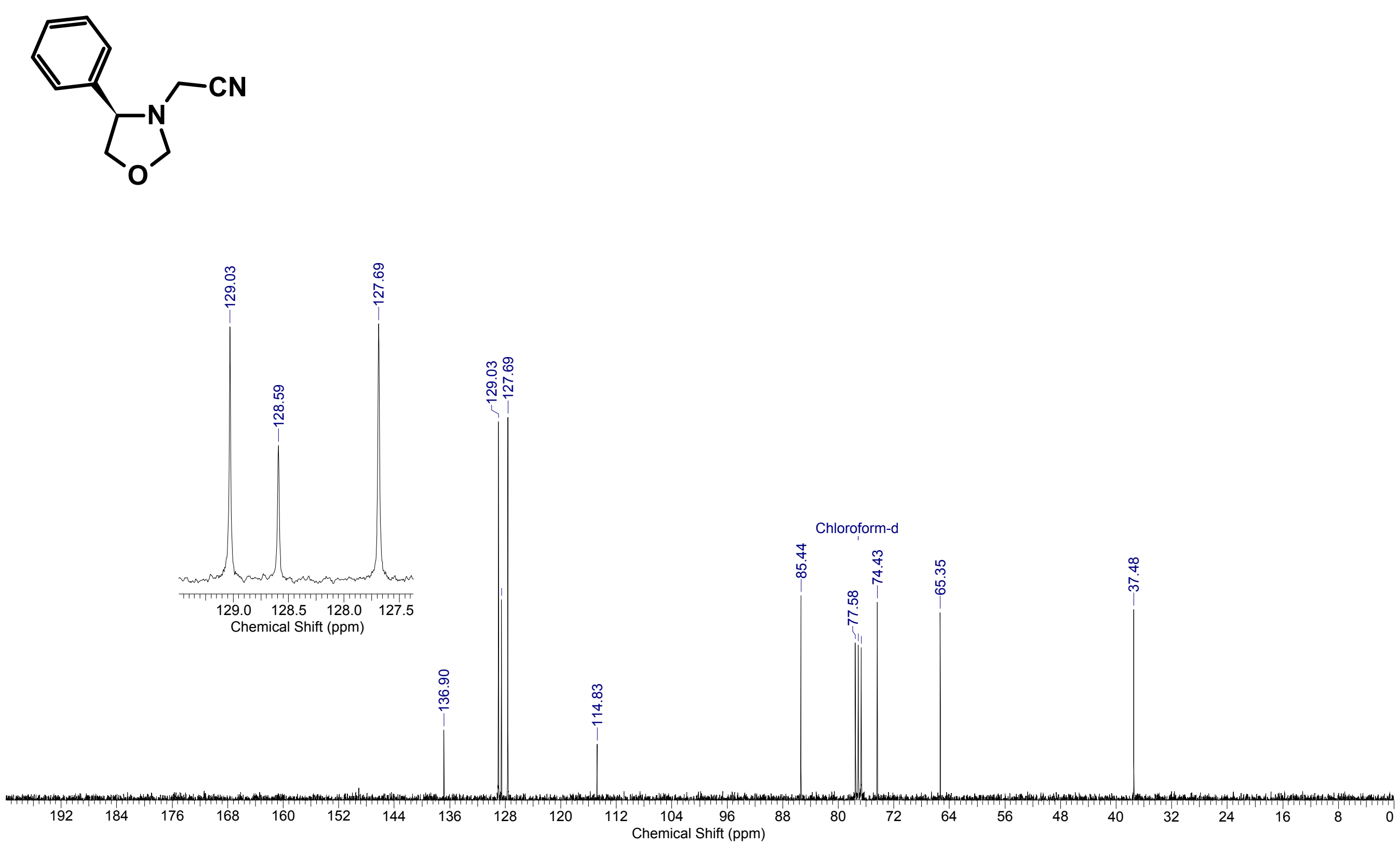


\section{Gas Chromatographic Conditions}

GC Column

FID Detector Air Flow

FID Detector $\mathrm{H}_{2}$ Flow

Column Flow

Oven Temperature

Detector Temperature

Inlet Temperature

Inlet Total Flow
Supelco 24348 Beta Dex 225, $30 \mathrm{~m}$ x $250 \mu \mathrm{m}$ x $0.25 \mu \mathrm{m}$ $400 \mathrm{~mL} / \mathrm{min}$

$30 \mathrm{~mL} / \mathrm{min}$

$1.5 \mathrm{~mL} / \mathrm{min}$

$150{ }^{\circ} \mathrm{C}$

$250{ }^{\circ} \mathrm{C}$

$240{ }^{\circ} \mathrm{C}$

$80.7 \mathrm{~mL} / \mathrm{min}$ 
(R) - i-Bu_morph
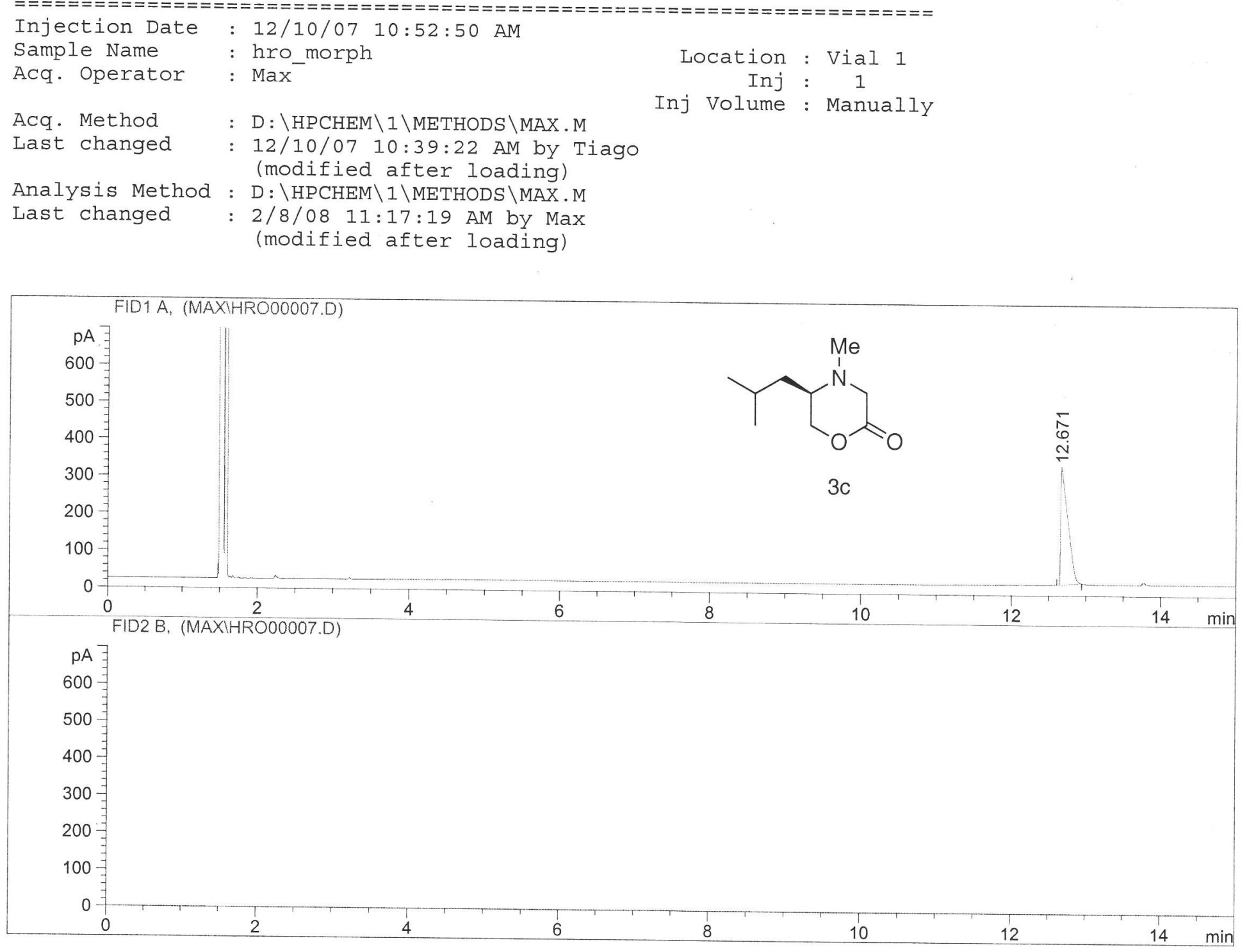

\section{Area Percent Report}

Sorted By

Multiplier

Dilution

Sample Amount
Signal

1.0000

1.0000

1.00000 [ng/ul] (not used in calc.)

Signal 1: FID1 A,

Peak RetTime Type width Area Height Area

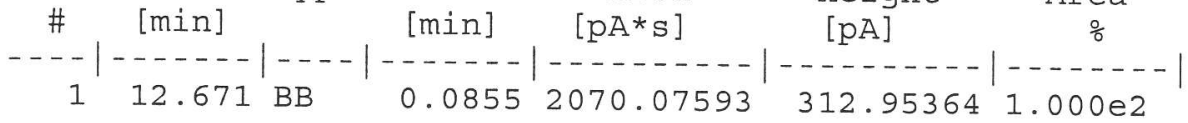

Totals :

$$
2070.07593 \quad 312.95364
$$

Results obtained with standard integrator! 
(S) -i-Bu_morph
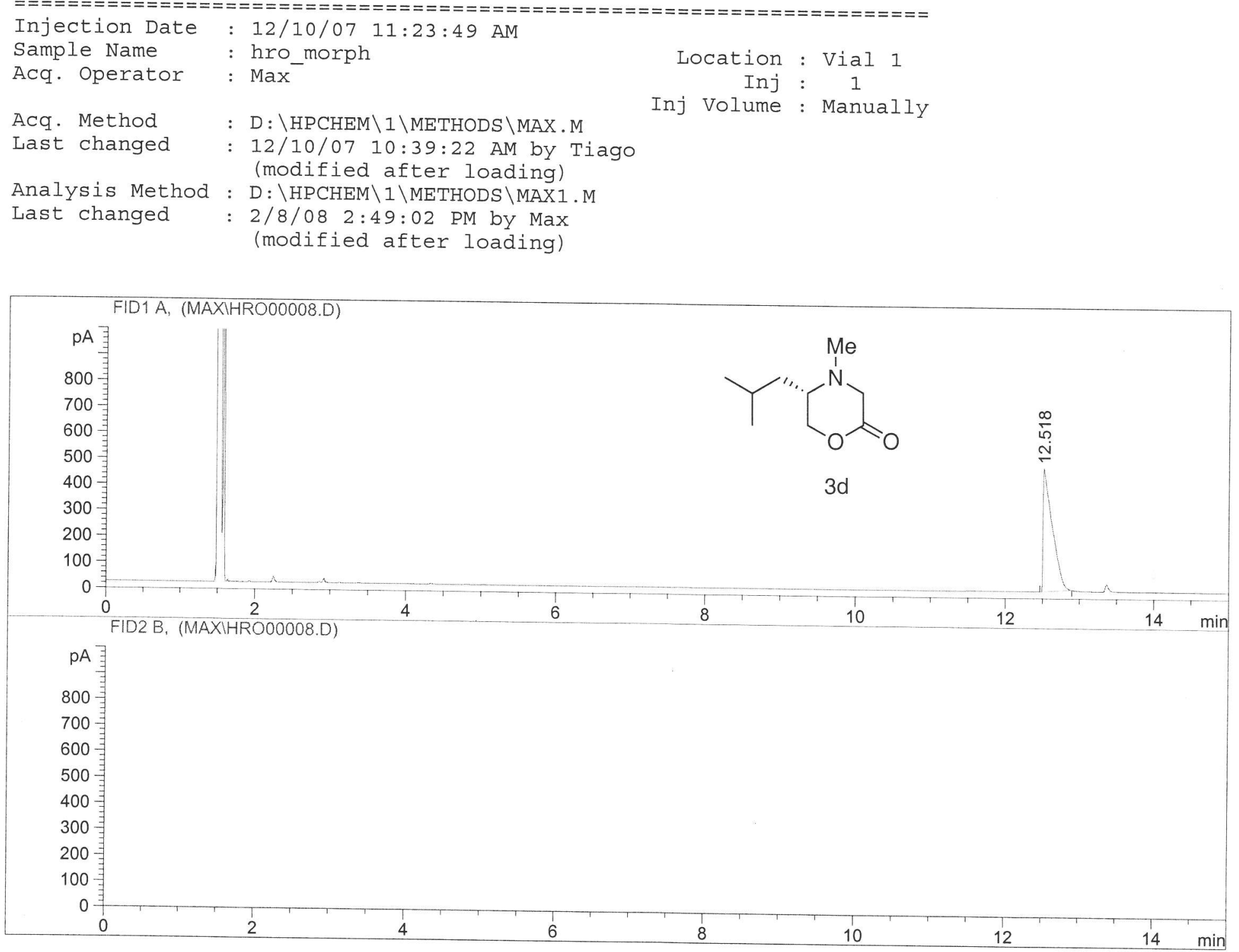

\section{Area Percent Report}

\section{Sorted By}

Multiplier

Dilution

Sample Amount

\section{Signal}

1.0000

1.0000

1.00000 [ng/ul] (not used in calc.)

Signal 1: FID1 A,

\begin{tabular}{|c|c|c|c|c|c|c|}
\hline $\begin{array}{c}\text { Peak } \\
\#\end{array}$ & $\begin{array}{l}\text { RetTime } \\
\text { [min] }\end{array}$ & Type & $\begin{array}{l}\text { Width } \\
\text { [min] }\end{array}$ & $\begin{array}{r}\text { Area } \\
{\left[\mathrm{pA}{ }^{*} \mathrm{~s}\right]}\end{array}$ & $\begin{array}{l}\text { Height } \\
{[\mathrm{pA}]}\end{array}$ & $\begin{array}{c}\text { Area } \\
\frac{\circ}{0}\end{array}$ \\
\hline & 12518 & Rס & -.---.- & - - - - & --------- & $-\cdots----$ \\
\hline 1 & 12.518 & BB & 0.1080 & 4076.79785 & 470.38477 & $1.000 e 2$ \\
\hline Tot & : & & & 4076.79785 & 470.38477 & \\
\hline
\end{tabular}

Results obtained with standard integrator! 
(S) -i-Bu_morph $+(\mathrm{R})-i-\mathrm{Bu}$ _morph

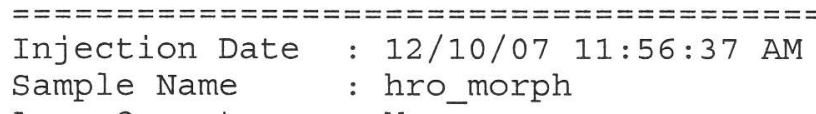

Acq. Operator : Max

Acq. Method : D: \HPCHEM \} \backslash \backslash \text { METHODS } \backslash \text { MAX.M }

Last changed : 12/10/07 10:39:22 AM by Tiago

(modified after loading)

Analysis Method : D: \HPCHEM $\backslash 1 \backslash$ METHODS $\backslash$ MAX $1 . M$

Last changed : 2/8/08 2:50:23 PM by Max

(modified after loading)

\section{Location : Vial 1}

Inj : 1

Inj Volume : Manually



\section{Area Percent Report}

Sorted By

Multiplier

Dilution

Sample Amount
Signal

1.0000

1.0000

1.00000

[ng/ul] (not used in calc.)

Signal 1: FID1 A,

\begin{tabular}{|c|c|c|c|c|c|c|}
\hline $\begin{array}{c}\text { Peak } \\
\quad \#\end{array}$ & $\begin{array}{l}\text { RetTime } \\
\text { [min] }\end{array}$ & Type & $\begin{array}{l}\text { Width } \\
\text { [min] }\end{array}$ & $\begin{array}{r}\text { Area } \\
{\left[\mathrm{pA}^{*} \mathrm{~s}\right]}\end{array}$ & $\begin{array}{l}\text { Height } \\
{[\mathrm{pA}]}\end{array}$ & $\begin{array}{c}\text { Area } \\
\frac{\circ}{6}\end{array}$ \\
\hline-- & & & & $-\cdots--\cdots$ & 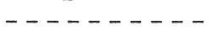 & $\ldots \ldots$ \\
\hline 1 & 12.543 & $\mathrm{BV}$ & 0.0406 & 1081.50354 & 371.61481 & 27.36085 \\
\hline 2 & 12.610 & VB & 0.1084 & 2871.23706 & 338.74570 & 72.63915 \\
\hline lotal & ls : & & & 3952.74060 & 710.36050 & \\
\hline
\end{tabular}

Results obtained with standard integrator! 